\title{
Genericity of mode-locking for quasiperiodically forced circle maps
}

\author{
J. Wang, Q. Zhou ${ }^{\dagger}$ and T. Jäger ${ }^{\ddagger}$
}

September 14, 2018

\begin{abstract}
We show that a generic quasiperiodically forced circle homeomorphism is mode-locked: the rotation number in the fibres is rationally related to the rotation number in the base and it is stable under small perturbations of the system. As a consequence, this implies that for a generic parameter family of quasiperiodically forced circle homeomorphisms satisfying a twist condition, the graph of the rotation number as a function of the parameter is a devil's staircase.
\end{abstract}

\section{Introduction}

One of the cornerstones of low-dimensional dynamics is Poincaré's rotation theory on the circle. Given an orientation-preserving circle homeomorphism $f: \mathbb{T}^{1} \rightarrow \mathbb{T}^{1}$, where $\mathbb{T}^{1}=\mathbb{R} / \mathbb{Z}$, and a lift $F: \mathbb{R} \rightarrow \mathbb{R}$, the rotation number of $F$ is defined as $\rho(F)=$ $\lim _{n \rightarrow \infty}\left(F^{n}(x)-x\right) / n$. This limit always exists and is independent of $x$ (e.g. 11, 2]). In this context, mode-locking means that the rotation number of $f$ is constant under small $\mathcal{C}^{0}$-perturbations. This can only happen if $\rho(f)$ is rational and occurs, for example, if $f$ has a stable fixed or periodic point. More generally, $f$ is mode-locked if and only if there exists a non-empty interval $I \subsetneq \mathbb{T}^{1}$ and some $p \in \mathbb{N}$ such that $f^{p}$ maps $I$ strictly inside itself (that is $f^{p}(\bar{I}) \subseteq \operatorname{int}(I)$ ). This is true for an open and dense set of orientation-preserving circle homeomorphisms, so that in this setting mode-locking is generic in a topological sense. Further, one may consider parameter families of circle homeomorphisms $\left(f_{\tau}\right)_{\tau \in \mathbb{T}^{1}}$ which satisfy a twist condition, that is, for all $x \in \mathbb{T}^{1}$ the mapping $\tau \mapsto f_{\tau}(x)$ is strictly order-preserving (that is, an orientation-preserving circle homeomorphism as well). Then as a consequence of the above, one obtains that in the topologically generic case the map $\tau \mapsto \rho\left(f_{\tau}\right)$ is locally constant on an open and dense set of parameters, so that its graph is a devil's staircase.

Our aim is to study the analogous phenomenon in the context of quasiperiodically forced (qpf) circle homeomorphisms. These are homeomorphisms of the two-dimensional torus, homotopic to the identity, with skew product structure

$$
f: \mathbb{T}^{2} \rightarrow \mathbb{T}^{2} \quad, \quad f(\theta, x)=\left(\theta+\omega, f_{\theta}(x)\right)
$$

Usually $\omega \in \mathbb{T}^{1}$ is supposed to be irrational - hence quasiperiodic forcing. However, for our purposes it will be convenient to include the rational case and to speak of periodically forced circle homeomorphisms in this case. The circle homeomorphisms $f_{\theta}: \mathbb{T}^{1} \rightarrow \mathbb{T}^{1}$ are called fibre maps.

There is one particular case that has to be mentioned in this context, which are quasiperiodic $\mathrm{SL}(2, \mathbb{R})$-cocycles. Via their projective action, these induce maps of the form (1.1) (e.g. 3]). For the special case of Schrödinger cocycles with potential $V(\theta)=$ $\lambda \cos (2 \pi \theta)$, the existence of a devil's staircase for the respective parameter families is equivalent to Cantor spectrum of the so called almost-Mathieu operator, which has been established in 4, 5, 6, 7]. For more general potentials $V$, Simon conjectured that the

\footnotetext{
*Department of Applied Mathematics, Nanjing University of Science and Technology, 210094 Nanjing, China. Email: jingwang018@gmail.com

$\dagger$ Department of Mathematics, Nanjing University, 210093 Nanjing, China. Email: qizhou@nju.edu.cn

$\ddagger$ Friedrich-Schiller-University Jena, Institute of Mathematics, 07743 Jena, Germany. Email: tobias.jaeger@uni-jena.de
} 
corresponding one-dimensional quasiperiodic Schrödinger operator generically has Cantor spectrum [8. We recall that one dimensional quasiperiodic Schrödinger operator is

$$
(H u)_{n}=u_{n+1}+u_{n-1}+V(\theta+n \omega) u_{n}
$$

with frequency $\omega \in \mathbb{T}^{d}(d \in \mathbb{N})$, and potential function $V \in C^{k}\left(\mathbb{T}^{d}, \mathbb{R}\right),(k \in \mathbb{N}, \infty, \omega)$. A number of results have been obtained on this problem, with the most studied case being the analytic one. Eliasson proved that for any fixed Diophantine frequency $\omega \in \mathbb{T}^{d}$, the quasiperiodic Schrödinger operator with generic small analytic potential has Cantor spectrum [9] Then, still in the analytic topology with $d=1$, Goldstein and Schlag proved that for generic $\omega \in \mathbb{R} \backslash \mathbb{Q}$, in the region of positive Lyapunov exponents (that is, for $V$ sufficiently large), the spectrum is again a Cantor set [10. In the $\mathcal{C}^{0}$-topology, AvilaBochi-Damanik proved Cantor spectrum for any fixed totally irrational vector $\omega \in \mathbb{T}^{d}$ and generic $V \in C^{0}\left(\mathbb{T}^{d}, \mathbb{R}\right)\left[11,12\right.$. In the case of $C^{k}$-topology $(1 \leq k \leq \infty$ or even in analytic category), Simon's conjecture is true for $d=1$ : one can prove that for generic $\omega \in \mathbb{R} \backslash \mathbb{Q}$ and generic $v \in C^{k}(\mathbb{T}, \mathbb{R})$, the spectrum of $(1.2)$ is a Cantor set 11 Results on the continuous-time case can be found in 9, 13.

Yet, for all these results the respective proofs depend strongly on the particular linear structure of $\mathrm{SL}(2, \mathbb{R})$-cocycles and can usually not be carried over directly to a more general nonlinear setting. A genuinely non-linear approach that is based on multiscale analysis and parameter exclusion in the spirit of Benedicks and Carleson [14 has been developed in [15, 16, 17. and applied to the problem of mode-locking both in the linear and the non-linear case [18, 19, 20]. This allows to provide effective criteria for the existence of a devil's staircase in explicit parameter families, although up to date only partial results are available for non-linear systems [20. In particular, there exist no explicit examples of non-linear systems for which a complete devil's staircase has been established.

Here, we take a different route and study the problem from the viewpoint of topological genericity, analogous to the results on generic Schrödinger operators mentioned above. We denote the class of maps of the form (1.1) by $\mathcal{F}$ and equip them with the metric $d(f, g)=\max \left\{d_{\mathcal{C}^{0}}(f, g), d_{\mathcal{C}^{0}}\left(f^{-1}, g^{-1}\right)\right\}$, where $d_{\mathcal{C}^{0}}$ denotes the canonical $\mathcal{C}^{0}$-distance. Note that thus $\mathcal{F}$ becomes a complete metric space, and hence a Baire space. The fibred rotation number is given by

$$
\rho(F, \theta)=\lim _{n \rightarrow \infty}\left(F_{\theta}^{n}(x)-x\right) / n,
$$

where $F: \mathbb{T}^{1} \times \mathbb{R} \rightarrow \mathbb{T}^{1} \times \mathbb{R}$ is a lift of $f$ and we write $F_{\theta}^{n}=F_{\theta+(n-1) \omega} \circ \ldots \circ F_{\theta}$ for the fibre maps of the iterates. Again, the limit in (1.3) always exists and is independent of $x \in \mathbb{R}$. Moreover, if $\omega$ is irrational, then it is also independent of $\theta$ (and will simply be denoted by $\rho(F)$ ) and the convergence is uniform on $\mathbb{T}^{2}[21,22$. The mechanism for mode-locking in qpf circle homeomorphisms in this situation is essentially the same as in the unforced case.

Theorem 1.1 (23]). A qpf circle homeomorphism $f$ is mode-locked if and only if there exists a topological annulus $\mathcal{A} \subseteq \mathbb{T}^{2}$ and some $p \in \mathbb{N}$ such that $f^{p}$ maps $\mathcal{A}$ strictly inside itself.

Remark 1.2. It can be shown that the annulus $\mathcal{A}$ is necessarily non-trivial in homotopy, so it 'wraps aroud the torus' in some direction. Moreover, in the terminology of [23], the intersection $\bigcap_{n \in \mathbb{N}} f^{n p}(\mathcal{A})$ is a $p$-periodic strip, and its existence forces the rotation numbers $\omega$ and $\rho(f)$ to be rationally related (that is, the equation $r \omega+s \rho(f)+t=0$ has a non-zero integer solution $(\hat{r}, \hat{s}, \hat{t}))$. We refer to [23] for details.

One advantage of this equivalent characterisation of mode-locking is that it can directly be extended to periodically forced circle homeomorphisms - where mode-locking is more difficult to define otherwise due to the independence of the fibres over disjoint orbits - and that it is obviously an open condition. It is thus perfectly adapted to address questions of genericity. We therefore make the following definition.

Definition 1.3. We say $f \in \mathcal{F}$ is mode-locked, if there exist a topological annulus $\mathcal{A} \subseteq \mathbb{T}^{2}$ and an integer $p$ such that $f^{p}$ maps $\mathcal{A}$ strictly inside itself.

1 To see this, one can first perturb the potential such that its related cocycle has positive Lyapunov exponent [12, and then perturb the frequency to obtain Cantor spectrum [10]. This proof has been pointed out to the second author by Avila. 
Our main result is the following.

Theorem 1.4. There exists an open and dense set $\mathcal{F}^{M L} \subseteq \mathcal{F}$ such that all $f \in \mathcal{F}^{M L}$ are mode-locked.

To the best knowledge of the authors, this result establishes the first nonlinear analogue to the Cantor spectrum results discussed above. It is important to note here that we work with the whole space $\mathcal{F}$, which allows to change the rotation number and use rational approximations for the proof. However, this also means that we obtain a statement mainly about Liouvillean rotation numbers in the base (which are topologically generic), and nothing can be said in this way about the Diophantine case (which is topologically meager). This becomes evident through the following statement. Given $\omega \in \mathbb{T}^{1}$, we let $\mathcal{F}_{\omega}=\{f \in \mathcal{F} \mid f$ has rotation number $\omega$ in the base $\}$ and equip this space with the induced $\mathcal{C}^{0}$-topology. Recall that a subset of a Baire space is called residual if it is a countable intersection of open and dense sets.

Corollary 1.5. There exists a residual set $\Omega \subseteq \mathbb{T}^{1}$ such that for all $\omega \in \Omega$ there is an open and dense set $\mathcal{F}_{\omega}^{M L}$ such that all $f \in \mathcal{F}_{\omega}^{M L}$ are mode-locked.

As a residual set, $\Omega$ will contain a residual subset of Liouvillean rotation numbers (since intersections of residual sets are residual), but does not necessarily contain any Diophantine numbers.

As in the one-dimensional case, we then turn to consider parameter families $\left(f_{\tau}\right)_{\tau \in \mathbb{T}^{1}}$ with a twist condition, by which we mean that for all $(\theta, x) \in \mathbb{T}^{2}$ the mapping $\tau \mapsto f_{\tau, \theta}(x)$ is strictly orientation-preserving. We denote the space of such families with fixed rotation number $\omega$ in the base by $\mathcal{P}_{\omega}$, let $\mathcal{P}=\bigcup_{\omega \in \mathbb{T}^{1}} \mathcal{P}_{\omega}$ and equip both sets with the respective $\mathcal{C}^{0}$-topology.

Theorem 1.6. (a) There exists a residual set $\mathcal{P}^{D S} \subseteq \mathcal{P}$ such that for all $\left(f_{\tau}\right)_{\tau \in \mathbb{T}^{1}} \in$ $\mathcal{P}^{D S}$ the graph of the function $\tau \mapsto \rho\left(f_{\tau}\right)$ is a devil's staircase.

(b) There exists a residual set $\Omega \subseteq \mathbb{T}^{1}$ with the property that for all $\omega \in \Omega$ there is a residual set $\mathcal{P}_{\omega}^{D S}$ such that for all $\left(f_{\tau}\right)_{\tau \in \mathbb{T}^{1}} \in \mathcal{P}_{\omega}^{D S}$ the graph of the function $\tau \mapsto \rho\left(f_{\tau}\right)$ is a devil's staircase.

Acknowledgments. We would like to thank the Alexander von Humboldt-Foundation, who has made this collaboration possible by supporting JW with a postdoc fellowship during her stay at the TU Dresden and the FSU Jena. TJ acknowledges support by the German Research Council (Emmy Noether grant Ja 1721/2-1 and Heisenberg grant Oe 538/6-1) and the EU-funded CRITICS network.

\section{Notation and preliminaries}

Given $x, y \in \mathbb{T}^{1}$, we denote by $[x, y]$ the positively oriented closed interval from $x$ to $y$, and use similar notation for open and half-open intervals. Maps defined on tori will usually be denoted by small letters (greek or latin) and their lifts by the respective captital letters. If $f: \mathbb{T}^{2} \rightarrow \mathbb{T}^{2}$ is a qpf circle homeomorphism, then we usually consider lifts $F: \mathbb{T}^{1} \times \mathbb{R} \rightarrow \mathbb{T}^{1} \times \mathbb{R}$ to the open annulus (and only occasionally lifts to the plane).

It will be convenient to introduce the following alternative notation for qpf circle homeomorphisms. Suppose $\varphi: \mathbb{T}^{2} \rightarrow \mathbb{T}^{1}$ is a continuous function such that for all $\theta \in \mathbb{T}^{1}$ the mapping $x \mapsto x+\varphi(\theta, x)$ defines an orientation-preserving circle homeomorphism. When $\varphi$ is differentiable, the latter means that $\partial_{x} \varphi(\theta, x) \geq-1$ for all $x$ and the set $\left\{x \in \mathbb{T}^{1} \mid \partial_{x} \varphi(\theta, x)=-1\right\}$ is nowhere dense. Here, we use $\partial_{\vartheta}$ to denote the derivative with respect to the variable $\vartheta$.

Given $\omega \in \mathbb{T}^{1}$, we can then define a map $(\omega, \varphi)$ of the form (1.1) by

$$
(\omega, \varphi):(\theta, x) \mapsto(\theta+\omega, x+\varphi(\theta, x)) .
$$

We denote the space of functions $\varphi: \mathbb{T}^{2} \rightarrow \mathbb{T}^{1}$ with the above properties by $\Xi$. Note that $\Xi$ is 'convex' in the following sense: If $\varphi, \hat{\varphi} \in \Xi$ and $\Phi, \hat{\Phi}: \mathbb{T}^{2} \rightarrow \mathbb{R}$ are lifts, then any convex combination of $\Phi$ and $\hat{\Phi}$ is again a lift of a function in $\Xi$. Thus, it is always possible to interpolate between two elements of $\Xi$ in a continuous way. In general, the path obtained by this interpolation depends on the choice of the lifts $\Phi$, $\hat{\Phi}$, which are only well-defined modulo additive integer constants. However, we will often have that $\hat{\varphi}$ is a small $\mathcal{C}^{0}$-perturbation of $\varphi$, that is, $\varepsilon=d_{\mathcal{C}^{0}}(\varphi, \hat{\varphi}) \ll 1$. In this case, there is a natural way 
to interpolate between $\varphi$ to $\hat{\varphi}$ within the $\varepsilon$-neighbourhood of the two functions, which is simply to chose lifts $\Phi, \hat{\Phi}$ with $d_{\mathcal{C}^{0}}(\Phi, \hat{\Phi})=d_{\mathcal{C}^{0}}(\varphi, \hat{\varphi})=\varepsilon$ for the convex combinations. In this situation, we will slightly abuse notation and write $t \varphi+(1-t) \hat{\varphi}$ without further explanation.

We will further need the following notation and conventions. Given a qpf circle homeomorphism $f$ with rotation number $\omega$ on the base, we let $\varphi_{f}(\theta, x)=f_{\theta}(x)-x$, so that $\left(\omega, \varphi_{f}\right)=f$. Further, given $\varphi \in \Xi$, we let $\varphi^{-1}=\varphi_{(\omega, \varphi)^{-1}}$ denote the respective translation function of the inverse of $(\omega, \varphi)$, so that $\left(-\omega, \varphi^{-1}\right)=(\omega, \varphi)^{-1}$. More generally, we denote by $\varphi^{q}$ the translation function associated to $f^{q}$, that is, $f^{q}=\left(q \omega, \varphi^{q}\right)$. If $(\omega, \varphi) \in \mathcal{F}$ and $\Phi: \mathbb{T}^{2} \rightarrow \mathbb{R}$ is a lift of $\varphi$, then $(\omega, \Phi): \mathbb{T}^{1} \times \mathbb{R} \rightarrow \mathbb{T}^{1} \times \mathbb{R},(\theta, x) \mapsto(\theta+\omega, x+\Phi(\theta, x))$ is a lift of $(\omega, \varphi)$. Thus, the rotation numbers $\rho(\omega, \Phi, \theta)$ (or simply $\rho(\omega, \Phi)$ in the quasiperiodic case) are defined by (1.3). Note that if $\omega=p / q$ is rational, with $p, q$ coprime integers, then $\rho(\omega, \Phi, \theta)$ is equal to $1 / q$ times the rotation number of $F_{\theta}^{q}$, where $F=(\omega, \Phi)$.

Further, we will need two basic statements concerning the behaviour of rotation numbers. Since both are folklore and follow from standard arguments, the proofs are omitted. The first statement concerns the continuity of the boundary of Arnold tongues.

Lemma 2.1. Suppose $\mathfrak{A}$ is a compact topological space and $\left(g_{\alpha, \tau}\right)_{\alpha \in \mathfrak{A}, \tau \in \mathbb{R}}$ is a twoparameter family of circle homeomorphisms (that is, $(\alpha, \tau, x) \mapsto g_{\alpha, \tau}(x)$ is continuous) with lifts $G_{\alpha, \tau}$ such that $(\alpha, \tau, x) \mapsto G_{\alpha, \tau}(x)$ is continuous and strictly monotone in $\tau$ for each $\alpha, x$ (twist condition).

Then for any $\rho \in \mathbb{R}$ there exist continuous functions $\tau_{\rho}^{-}, \tau_{\rho}^{+}: \mathfrak{A} \rightarrow \mathbb{R}$ such that

$$
\left\{(\alpha, \tau) \in \mathfrak{A} \times \mathbb{R} \mid \rho\left(G_{\alpha, \tau}\right)=\rho\right\}=\left\{(\alpha, \tau) \in \mathfrak{A} \times \mathbb{R} \mid \tau_{\rho}^{-}(\alpha) \leq \tau \leq \tau_{\rho}^{+}(\alpha)\right\} .
$$

The analogue statement even holds in the qpf case, see 23 .

The second lemma concerns the uniform convergence of the fibred rotation number in parameter families.

Lemma 2.2. Suppose that $\mathcal{T}$ is a compact topological space, $\omega \in \mathbb{T}^{1}$ is irrational, $\left(f_{\tau}=\left(\omega, \varphi_{\tau}\right)\right)_{\tau \in \mathcal{T}} \subseteq \mathcal{F}$ is a continuous family of qpf circle homeomorphisms with lifts $\left(F_{\tau}\right)_{\tau \in \mathcal{T}},\left(f_{n, \tau}=\left(\omega_{n}, \varphi_{n, \tau}\right)\right)_{\tau \in \mathcal{T}, n \in \mathbb{N}}$ is a sequence of such parameter families with lifts $\left(F_{n, \tau}\right)_{\tau \in \mathcal{T}, n \in \mathbb{N}}$ and $\lim _{n \rightarrow \infty} F_{n, \tau}=F_{\tau}$ for all $\tau \in \mathcal{T}$. Then for all $\varepsilon>0$ there exist $m_{0}, n_{0} \in \mathbb{N}$ such that for $m \geq m_{0}$ and $n \geq n_{0}$ and all $\tau \in \mathcal{T}, \theta \in \mathbb{T}^{1}$ we have

$$
\left|\frac{1}{m}\left(F_{n, \tau, \theta}^{m}(x)-x\right)-\rho\left(F_{\tau}\right)\right|<\varepsilon .
$$

In particular, $\rho\left(F_{n, \tau}, \theta\right) \rightarrow \rho\left(F_{\tau}\right)$ uniformly on $\mathbb{T}^{1} \times \mathcal{T}$ as $n \rightarrow \infty$.

\section{$3 \quad$ Strategy of the proof}

Our key result, which entails the others rather easily (as explained in Section 5), is Theorem 1.4 For its proof, we only have to show that mode-locked qpf circle homeomorphisms $(\omega, \varphi)$ are dense in $\mathcal{F}$, since the openness is obvious from Definition 1.3 In order to do so, we fix an initial map $f=(\omega, \varphi) \in \mathcal{F}$ and perturb it in several steps to obtain the existence of an annulus that is mapped strictly inside itself by some iterate. This will be done in four main steps.

Step 1 First, we replace the rotation number $\omega$ in the base by some rational numer $p / q$ to obtain a periodically forced circle homeomorphism. By doing so, we may lose the uniqueness of the rotation number. However, by continuity ( see Lemma 2.2), the rotation numbers $\rho(p / q, \Phi, \theta)$ are still close to the original $\rho(\omega, \Phi)$. We then show that by means of a small modification of $\varphi$ we can ensure that the rotation numbers on the fibres are all the same and take a rational value. This is done by an indirect argument: we assume that no system in a neighbourhood of $(\omega, \varphi)$ is mode-locked (so that mode-locking is not dense) and build the required perturbation only under this assumption (since otherwise there is nothing to prove anyway).

Step 2 Once this is achieved, it follows from the standard theory of orientation-preserving circle homeomorphisms that all the fibre maps $f_{\theta}^{q}$ have a fixed point. We then proceed to show that $\varphi$ can be perturbed in such a way that all the fibre maps $f_{\theta}^{q}$ are mode-locked. 
However, this does not yet imply that $f$ itself is mode-locked in the sense of Definition 1.3 - a forward invariant or periodic annulus $\mathcal{A}$ does not yet have to exist for this perturbation. This happens, for example, if $\omega=p / q$ and the complement of set $B=\left\{(\theta, x) \in \mathbb{T}^{2} \mid \Phi^{q}(\theta, x)=0\right\}$ is inessential 2 In this case, the boundary of any homotopically non-trivial annulus $\mathcal{A} \subseteq \mathbb{T}^{2}$ has to intersect $B$, and can therefore not be mapped into its own interior by any iterate of $f$ (since points of $B$ are fixed under $f^{q}$.

What we obtain so far, though, is the fact that the sets $B^{-}=\left\{(\theta, x) \in \mathbb{T}^{2} \mid\right.$ $\left.\Phi^{q}(\theta, x)<0\right\}$ and $B^{+}=\left\{(\theta, x) \in \mathbb{T}^{2} \mid \Phi^{q}(\theta, x)>0\right\}$ both project to all of $\mathbb{T}^{1}$.

Step 3 We then perturb $\varphi$ to ensure that both $B^{-}$and $B^{+}$consist of only finitely many connected components with positive distance to each other, and have some further good properties.

Step 4 The final step consists in showing that, in the situation obtained in Step 3, there exists a small neighbourhood $B_{\varepsilon}(p / q)$ such that for every $\omega^{\prime} \in B_{\varepsilon}(p / q) \backslash\{p / q\}$ the system $\left(\omega^{\prime}, \varphi\right)$ is mode-locked in the sense of Definition 1.3

While the arguments in Steps 1-3 are rather standard, it is the last step which contains the main new geometric idea of our proof and depends crucially on the topological characterisation of mode-locking in Definition 1.3 It is also this part in which the difference to the linear-projective case of quasiperiodic $\mathrm{SL}(2, \mathbb{R})$-cocycles becomes most apparent. What happens is that we have to deal with the fact that the desired annulus $\mathcal{A}$ may be of any homotopy type, whereas in the linear case the corresponding forward invariant annuli (which correspond to invariant cone fields for the cocycle) can only have homotopy types of the form $(1, k)$ with $k \in \mathbb{Z}$ (that is, they wrap around the torus exactly once in the horizontal direction).

\section{Proof of Theorem 1.4}

As mentioned, we fix $f=(\omega, \varphi) \in \mathcal{F}$ with lift $F=(\omega, \Phi)$ and show that there exists an arbitrarily small perturbation of $f$ in $\mathcal{F}$ that exhibits mode-locking, following the steps outlined in the previous section. The following proposition corresponds to Step 1.

Proposition 4.1. Suppose that $(\omega, \varphi) \in \mathcal{F}$ and there exists a neighbourhood $B_{\eta}(\omega, \varphi)$ in $\mathcal{F}$ such that no $f \in B_{\eta}(\omega, \varphi)$ is mode-locked. Then there exists $(\tilde{\omega}, \tilde{\varphi}) \in \mathcal{F}$ such that

- $(\tilde{\omega}, \tilde{\varphi})$ is an arbitrarily small perturbation of $(\omega, \varphi)$;

- $\tilde{\omega}=p / q$, where $p, q$ are coprime integers;

- there exists $m_{0} \in \mathbb{N}$ such that $\rho(\tilde{\omega}, \tilde{\Phi}, \theta)=m_{0} / q$ for all $\theta \in \mathbb{T}^{1}$.

Proof. Fix $0<\varepsilon<1$. We may assume that $\omega$ is irrational, so that $(\omega, \Phi)$ has a unique rotation number. (Otherwise, we just replace $\omega$ by some nearby irrational number). Moreover, since systems in a neighbourhood of $(\omega, \varphi)$ are not mode-locked, there exists $\delta>0$ such that

$$
\rho(\omega, \Phi-\varepsilon)+\delta<\rho(\omega, \Phi)<\rho(\omega, \Phi+\varepsilon)-\delta .
$$

We now replace $\omega$ by $\tilde{\omega}=p / q$ with $1 / q<\delta / 2$. Then Lemma 2.2 implies that if $\tilde{\omega}$ is chosen sufficiently close to $\omega$, then

$$
|\rho(\tilde{\omega}, \Phi+\tau, \theta)-\rho(\omega, \Phi+\tau)|<\delta / 2
$$

for all $\tau \in[-1,1]$. Fix $m_{0} \in \mathbb{N}$ such that $\left|\rho(\omega, \Phi)-m_{0} / q\right|<\delta / 2$. By Lemma 2.1, there exists a continuous $1 / q$-periodic function $\tau^{+}: \mathbb{T}^{1} \rightarrow \mathbb{R}$ such that $\rho\left(\tilde{\omega}, \Phi+\tau^{+}, \theta\right)=m_{0} / q$ for all $\theta \in \mathbb{T}^{1}$. In order to see this, apply Lemma 2.1 with $\mathfrak{A}=\mathbb{T}^{1}, \alpha=\theta, g_{\tau, \theta}=$ $R_{\tau} \circ f_{\theta+(q-1) \tilde{\omega}} \circ \ldots \circ R_{\tau} \circ f_{\theta}$ and $\rho=m_{0}$ and note that since the maps $g_{\tau, \theta}$ and $g_{\tau, \theta+i p / q}$ are conjugate for all $i=1, \ldots, q-1$, the resulting functions $\tau^{ \pm}=\tau_{m_{0}}^{ \pm}$are $1 / q$-periodic. By

\footnotetext{
${ }^{2}$ An open set $U \subseteq \mathbb{T}^{2}$ is called essential if it contains some homotopically non-trivial closed curve $\gamma \subseteq U$, and it is called inessential otherwise. It is called doubly essential, if it contains two homotopically non-trivial closed curves of different homotopy types. An arbitrary set $A$ is called essential (doubly essential), if any open neighbourhood is essential (doubly essential).
} 
definition, we also have $\rho\left(G_{\tau, \theta}\right)=q \rho(\tilde{\omega}, \Phi+\tau, \theta)$, where $G_{\tau, \theta}=R_{\tau} \circ F_{\theta+(q-1) \tilde{\omega}} \circ \ldots \circ R_{\tau} \circ F_{\theta}$, so $\rho\left(\tilde{\omega}, \Phi+\tau^{+}, \theta\right)=m_{0} / q$.

We claim that $\left|\tau^{+}\right|<\varepsilon$. This holds since

$$
\rho\left(G_{\varepsilon, \theta}\right)=q \rho(\tilde{\omega}, \Phi+\varepsilon, \theta) \stackrel{4.2}{>} q \rho(\omega, \Phi+\varepsilon)-q \delta / 2 \stackrel{4.1}{>} q \rho(\omega, \Phi)+q \delta / 2>m_{0},
$$

and likewise $\rho\left(G_{-\varepsilon, \theta}\right)<m_{0}$ for all $\theta \in \mathbb{T}^{1}$.

In conclusion, since $\varepsilon>0$ was arbitrary, we obtain the required perturbation $(\tilde{\omega}, \tilde{\varphi})=$ $\left(p / q, \varphi+\tau^{+}\right)$.

As in Section 3 if $\omega=p / q$ is rational with $p, q$ coprime and $(\omega, \varphi)$ satisfies the assertions of Proposition 4.1 we let

$$
\begin{aligned}
& B(\varphi)=\left\{(\theta, x) \in \mathbb{T}^{2} \mid \Phi^{q}(\theta, x)=0\right\}, \\
& B^{-}(\varphi)=\left\{(\theta, x) \in \mathbb{T}^{2} \mid \Phi^{q}(\theta, x)<0\right\}, \\
& B^{+}(\varphi)=\left\{(\theta, x) \in \mathbb{T}^{2} \mid \Phi^{q}(\theta, x)>0\right\},
\end{aligned}
$$

where $\Phi^{q}$ is the lift of $\varphi^{q}$ such that $\left\{x \in \mathbb{T}^{1} \mid \Phi^{q}(\theta, x)=0\right\} \neq \emptyset$ for all $\theta \in \mathbb{T}^{1}$. Then the following proposition takes care of Step 2.

Proposition 4.2. Suppose $(\omega, \varphi) \in \mathcal{F}$ and there exists a neighborhood $B_{\eta}(\omega, \varphi)$ in $\mathcal{F}$ such that no $f \in B_{\eta}(\omega, \varphi)$ is mode-locked. Then there exists $\tilde{f}=(\tilde{\omega}, \tilde{\varphi}) \in \mathcal{F}$ such that

- $(\tilde{\omega}, \tilde{\varphi})$ satisfies the assertions of Proposition 4.1 ;

- $\pi_{1}\left(B^{+}(\tilde{\varphi})\right)=\pi_{1}\left(B^{-}(\tilde{\varphi})\right)=\mathbb{T}^{1}$.

Note that the last property means that all the fibre maps $\tilde{f}_{\theta}^{q}$ of $\tilde{f}^{q}$ are mode-locked (as maps of the circle).

For the proof, we need two auxiliary lemmas that address the following technical issue: If we perturb the map $f$ to obtain certain qualitative properties of the fibre maps $f_{\theta}^{q}$, we need to take into account that these fibre maps are not independent. More precisely, if $\theta, \theta^{\prime}$ belong to the same periodic orbit in the base, both $f_{\theta}^{q}$ and $f_{\theta^{\prime}}^{q}$ result from the composition of the same family of fibre maps $f_{\theta+\frac{i}{q}}$, and only differ in the order of composition. Most importantly, they are conjugate to each other. This is the content of the following

Lemma 4.3. Suppose $\omega=p / q$ is rational, where $p, q$ are coprime integers. Then for any $\theta \in \mathbb{T}^{1}, j=1,2, \ldots, q-1$, the fibre maps $f_{\theta}^{q}$ and $f_{\theta+j / q}^{q}$ are conjugate.

Proof. Since $p, q$ are coprime, there exist unique $s, k \in \mathbb{N}$, such that $s p-k q=1$, which means that for any $j=1,2, \ldots, q-1$, we have $\frac{p}{q} \cdot j s=j k+\frac{j}{q}$. Further $f_{\theta}^{j s+q}=$ $f_{\theta+\frac{p}{q} \cdot j s}^{q} \circ f_{\theta}^{j s}=f_{\theta}^{j s} \circ f_{\theta}^{q}$. Together with the fact that $f_{\theta+n}=f_{\theta}$ for all $n \in \mathbb{N}$, this implies that

$$
f_{\theta+\frac{j}{q}}^{q}=f_{\theta+\frac{p}{q} \cdot j s}^{q}=f_{\theta}^{j s} \circ f_{\theta}^{q} \circ\left(f_{\theta}^{j s}\right)^{-1} .
$$

The next lemma allows to restrict to an interval $[\theta, \theta+1 / q)$ when perturbing $(p / q, \varphi)$.

Lemma 4.4. Let $\omega=p / q$ be rational with $p, q \in \mathbb{N}$ coprime, and $I \subseteq \mathbb{T}^{1}$ be a half open interval with $|I| \leq 1 / q$. Suppose $\psi \in \Xi$ and $\psi(\theta, \cdot)=\varphi^{q}(\theta, \cdot)$ for $\theta \in \partial I$. Then there exists $(\omega, \tilde{\varphi}) \in \mathcal{F}$ such that for any $\theta \in I$, we have $\tilde{\varphi}^{q}(\theta, \cdot)=\psi(\theta, \cdot)$.

Moreover, $(\omega, \tilde{\varphi})$ can be chosen as an arbitrary small perturbation of $(\omega, \varphi)$ provided that $\psi(\theta, \cdot)$ is sufficiently close to $\varphi^{q}(\theta, \cdot)$ for all $\theta \in I$.

Proof. Let $f=(\omega, \varphi)$ and $g=(\omega, \psi)$ and define

$$
\tilde{\psi}(\theta, x)= \begin{cases}\psi(\theta, x) & \text { for } \theta \in I, x \in \mathbb{T}^{1} \\ \varphi^{q}(\theta, x) & \text { for } \theta \in \mathbb{T}^{1} \backslash I, x \in \mathbb{T}^{1} .\end{cases}
$$

Then $\tilde{\psi} \in \Xi$ and hence $\tilde{g}=(\omega, \tilde{\psi}) \in \mathcal{F}$. For $(\theta, x) \in \mathbb{T}^{2}$, we let $\tilde{\varphi}(\theta, x)=\varphi_{\tilde{f}}(\theta, x)$ with $\tilde{f}_{\theta}(x)=f_{\theta}^{-(q-1)} \circ \tilde{g}_{\theta}(x)$. Then $\tilde{f}=(\omega, \tilde{\varphi}) \in \mathcal{F}$ and we obtain

$$
\tilde{f}_{\theta}(x)= \begin{cases}f_{\theta}^{-(q-1)} \circ g_{\theta}(x) & \text { for } \theta \in I, x \in \mathbb{T}^{1} \\ f_{\theta}(x) & \text { for } \theta \in \mathbb{T}^{1} \backslash I, x \in \mathbb{T}^{1} .\end{cases}
$$


Due to the fact that for any $\theta \in I, l=1,2, \ldots, q-1$, we have $\theta+l \frac{p}{q}(\bmod 1) \notin I$, we obtain

$$
\tilde{f}_{\theta}^{q}=\tilde{f}_{\theta+p / q}^{q-1} \circ \tilde{f}_{\theta}=f_{\theta+p / q}^{q-1} \circ f_{\theta}^{-(q-1)} \circ g_{\theta}=g_{\theta}, \forall \theta \in I
$$

and hence $\tilde{\varphi}^{q}(\theta, \cdot)=\psi(\theta, \cdot)$ for any $\theta \in I$ as required.

Moreover, by (4.3), for $\theta \in I$, we have $\tilde{f}_{\theta}-f_{\theta}=f_{\theta}^{-(q-1)} \circ g_{\theta}-f_{\theta}^{-(q-1)} \circ f_{\theta}^{q}$, which implies that $\tilde{f}$ is arbitrarily close to $f$ if $\psi(\theta, \cdot)$ is sufficiently close to $\varphi^{q}(\theta, \cdot)$ for all $\theta \in I$.

Proof of Proposition 4.2. Fix $\varepsilon>0$. Without loss of generality, we may assume that $(\omega, \varphi)$ satisfies the assertions of Proposition 4.1. For $\theta \in \mathbb{T}^{1}$, we let

$$
\begin{aligned}
A(\theta, \Phi) & =\left\{x \in \mathbb{T}^{1} \mid \Phi^{q}(\theta, x)=0\right\}, \\
A^{+}(\theta, \Phi) & =\left\{x \in \mathbb{T}^{1} \mid \Phi^{q}(\theta, x)>0\right\}, \\
A^{-}(\theta, \Phi) & =\left\{x \in \mathbb{T}^{1} \mid \Phi^{q}(\theta, x)<0\right\} .
\end{aligned}
$$

It is apparent that $\pi_{1}\left(B^{+}(\varphi)\right)=\pi_{1}\left(B^{-}(\varphi)\right)=\mathbb{T}^{1}$ is equivalent to $A^{+}(\theta, \Phi) \neq \emptyset \neq$ $A^{-}(\theta, \Phi)$ for all $\theta \in \mathbb{T}^{1}$. Note that this also implies $A(\theta, \Phi) \neq \emptyset$ for all $\theta \in \mathbb{T}^{1}$ by continuity. We proceed in two steps and start by showing that there exists $\hat{\varphi} \in B_{\varepsilon / 2}(\varphi) \cap \Xi$ such that $A(\theta, \hat{\Phi})$ is neither empty nor equal to $\mathbb{T}^{1}$ for all $\theta \in \mathbb{T}^{1}$, which implies that $A^{+}(\theta, \hat{\Phi}) \cup A^{-}(\theta, \hat{\Phi}) \neq \emptyset$ for all $\theta \in \mathbb{T}^{1}$ and hence $\pi_{1}\left(B^{-}(\hat{\varphi})\right) \cup \pi_{1}\left(B^{+}(\hat{\varphi})\right)=\mathbb{T}^{1}$. Secondly, we show that $\hat{\varphi}$ can be modified further and there exists $\tilde{\varphi} \in B_{\varepsilon / 2}(\hat{\varphi}) \cap \Xi$ such that $\pi_{1}\left(B^{-}(\tilde{\varphi})\right)=\pi_{1}\left(B^{+}(\tilde{\varphi})\right)=\mathbb{T}^{1}$.

We may assume that there is some open interval of $\theta \in \mathbb{T}^{1}$ for which $A(\theta, \Phi) \neq \mathbb{T}^{1}$, that is, $f_{\theta}^{q} \neq \mathrm{Id}_{\mathbb{T}^{1}}$. Otherwise, using Lemma 4.4 we can perturb $(\omega, \varphi)$ in a small interval on the base such that $A(\theta, \Phi) \neq \mathbb{T}^{1}$ for $\theta$ in the interior of this interval and the perturbed map still satisfies the assertions of Proposition 4.1 Without loss of generality, we assume that $\theta=0$ lies in this interval and hence $A(\theta, \Phi) \neq \mathbb{T}^{1}$ for $\theta=0$. Then by Lemma 4.3 . $A(\theta, \Phi) \neq \mathbb{T}^{1}$ for $\theta=1 / q$, i.e. $f_{1 / q}^{q} \neq \operatorname{Id}_{\mathbb{T}^{1}}$. Let

$$
\Theta=\left\{\theta \in[0,1 / q] \mid f_{\theta}^{q}=\operatorname{Id}_{\mathbb{T}^{1}}\right\} .
$$

Then, since $\Theta$ is compact and does not contain 0 and $1 / q$, we have that for any $\delta>0$ there exist finite number of disjoint closed intervals $J_{1}, \ldots, J_{M}$ and disjoint open intervals $U_{1}, \ldots, U_{M}$, such that $J_{i} \subseteq U_{i} \subseteq(0,1 / q),(i=1, \ldots, M), \Theta \subseteq \cup_{i=1}^{M} J_{i}$, and for all $\theta \in$ $\cup_{i=1}^{M} \operatorname{cl}\left(U_{i}\right)$ and $x \in \mathbb{T}^{1}$, we have $\left|\varphi^{q}(\theta, x)\right|<\delta$. Then for each $U_{i},(i=1, \ldots, M)$, there exists a continuous function $\eta_{i}: \operatorname{cl}\left(U_{i}\right) \times \mathbb{T}^{1} \rightarrow \mathbb{T}^{1}$ which is differentiable with respect to $x$ and satisfies

- $\left|\eta_{i}(\theta, x)\right|<\delta, \forall \theta \in \operatorname{cl}\left(U_{i}\right), x \in \mathbb{T}^{1}$, and $\partial_{x} \eta_{i}(\theta, x)>-1, \forall \theta \in U_{i}, x \in \mathbb{T}^{1}$;

- for any $\theta \in U_{i}$, there exist $x_{1}, x_{2} \in \mathbb{T}^{1}$ such that $\eta_{i}\left(\theta, x_{1}\right)=0$ and $\eta_{i}\left(\theta, x_{2}\right) \neq 0$;

- $\eta_{i}(\theta, x)=\varphi^{q}(\theta, x)$ for $\theta \in \partial U_{i}, x \in \mathbb{T}^{1}$.

Let

$$
\psi_{1}(\theta, x)=\left\{\begin{array}{ll}
\eta_{i}(\theta, x) & \text { for } \theta \in U_{i} \\
\varphi^{q}(\theta, x) & \text { for } \theta \in \mathbb{T}^{1} \backslash \cup_{i=1}^{M} U_{i}
\end{array} .\right.
$$

Then $\psi_{1} \in \Xi$, and for all $(\theta, x) \in \mathbb{T}^{2}$, we have $\left|\psi_{1}(\theta, x)-\varphi^{q}(\theta, x)\right|<2 \delta$. Applying Lemma 4.4 with $I=[0,1 / q), \psi=\psi_{1}$, there exists $\hat{\varphi} \in \Xi$, such that $\hat{\varphi}^{q}(\theta, x)=\psi_{1}(\theta, x)$ for all $\theta \in[0,1 / q), x \in \mathbb{T}^{1}$. Due to the choice of $\eta_{i}$, this implies that $A(\theta, \hat{\Phi})$ is neither empty nor $\mathbb{T}^{1}$ for $\theta \in[0,1 / q)$. Then by Lemma 4.3. this holds for all $\theta \in \mathbb{T}^{1}$. Moreover, by Lemma 4.4 if we choose $\delta$ small enough we can ensure that $\hat{f}=(\omega, \hat{\varphi}) \in B_{\varepsilon / 2}(\omega, \varphi)$. This completes the first part of the proof.

Now, let $J, J^{\prime}$ be two closed intervals of $\mathbb{T}^{1}$ with $|J|,\left|J^{\prime}\right|<1 / q$ and

$$
\bigcup_{i=0}^{q-1}(J+i \omega) \cup\left(J^{\prime}+i \omega\right)=\mathbb{T}^{1}
$$

Choose two left closed right open intervals $\tilde{J}, \tilde{J}^{\prime} \subseteq \mathbb{T}^{1}$ with $|\tilde{J}|=\left|\tilde{J}^{\prime}\right|=1 / q$, such that $J \subseteq \operatorname{int}(\tilde{J}), J^{\prime} \subseteq \operatorname{int}\left(\tilde{J}^{\prime}\right)$. For $\theta \in \mathbb{T}^{1}$, denote

$$
\gamma_{\hat{\Phi}}^{+}(\theta)=\sup _{x \in \mathbb{T}^{1}} \hat{\Phi}^{q}(\theta, x), \text { and } \gamma_{\hat{\Phi}}^{-}(\theta)=-\inf _{x \in \mathbb{T}^{1}} \hat{\Phi}^{q}(\theta, x) \text {. }
$$


Then there exists $C>0$ such that for any $\theta \in \mathbb{T}^{1}$, we have

$$
\gamma_{\hat{\Phi}}^{ \pm}(\theta) \in[0, C] \quad \text { and } \quad \gamma_{\hat{\Phi}}^{+}(\theta)+\gamma_{\hat{\Phi}}^{-}(\theta)>0
$$

where the last inequality follows from the fact that $\pi_{1}\left(B^{-}(\hat{\varphi})\right) \cup \pi_{1}\left(B^{+}(\hat{\varphi})\right)=\mathbb{T}^{1}$. Moreover, there exists a continuous function $\lambda: \mathbb{T}^{1} \rightarrow[0,1]$ supported on $\tilde{J}$ and $\lambda_{\mid J}=1$. For $0<\zeta<1 / 2$, we let

$$
\psi_{2}(\theta, x)=\hat{\varphi}^{q}(\theta, x)-\zeta \lambda(\theta) \gamma_{\hat{\Phi}}^{+}(\theta)+\zeta \lambda(\theta) \gamma_{\hat{\Phi}}^{-}(\theta)
$$

and apply Lemma 4.4 with $\psi=\psi_{2}, I=\tilde{J}$ and $\varphi=\hat{\varphi}$. Then there exists $\bar{\varphi} \in \Xi$, such that $\bar{\varphi}^{q}(\theta, x)=\psi_{2}(\theta, x)$ for $\theta \in \tilde{J}, x \in \mathbb{T}^{1}$. Therefore, for $\theta \in J$, we obtain that

$$
\gamma_{\bar{\Phi}}^{+}(\theta)=(1-\zeta) \gamma_{\hat{\Phi}}^{+}(\theta)+\zeta \gamma_{\hat{\Phi}}^{-}(\theta)>0 .
$$

In the same way, we obtain $\gamma_{\overline{\bar{\Phi}}}^{-}(\theta)>0$ for $\theta \in J$. Thus, we have $\gamma_{\bar{\Phi}}^{ \pm}(\theta)>0$ for all $\theta \in \cup_{i=0}^{q-1} J+i \omega$ by Lemma 4.3 Moreover, as $d_{\mathcal{C}^{0}}\left(\psi_{2}, \hat{\varphi}^{q}\right) \leq 2 C \zeta$, we can ensure that $d_{\mathcal{C}^{0}}(\bar{\varphi}, \hat{\varphi})<\varepsilon / 4$ by choosing $\zeta$ is small enough, see Lemma 4.4

As for $J^{\prime}, \tilde{J}^{\prime}$, similarly as before, we let

$$
\psi_{3}(\theta, x)=\bar{\varphi}^{q}(\theta, x)-\tilde{\zeta} \tilde{\lambda}(\theta) \gamma_{\bar{\Phi}}^{+}(\theta)+\tilde{\zeta} \tilde{\lambda}(\theta) \gamma_{\bar{\Phi}}^{-}(\theta)
$$

where $0<\tilde{\zeta}<1 / 2, \tilde{\lambda}: \mathbb{T}^{1} \rightarrow[0,1]$ is continuous and supported on $\tilde{J}^{\prime}$ and $\tilde{\lambda}_{\mid J^{\prime}}=1$. Applying Lemma 4.4 once more, with $\psi=\psi_{3}, I=\tilde{J}^{\prime}, \varphi=\bar{\varphi}$, we obtain a $\tilde{\varphi} \in \Xi$ such that $\tilde{\varphi}^{q}(\theta, x)=\psi_{3}(\theta, x)$ for $\theta \in \tilde{J}^{\prime}, x \in \mathbb{T}^{1}$. Then, we have

$$
\gamma_{\tilde{\Phi}}^{ \pm}(\theta)>0
$$

for all $\theta \in \bigcup_{i=0}^{q-1} J^{\prime}+i \omega$. It remains to show that 4.5 now holds for all $\theta \in \mathbb{T}^{1}$. To that end, suppose $\theta \in \tilde{J}^{\prime} \backslash \bigcup_{i=0}^{q-1} J^{\prime}+i \omega$. Then by the choice of $J, J^{\prime}, \tilde{J}^{\prime}$, we have that $\theta \in \cup_{i=0}^{q-1}(J+i \omega)$. Therefore,

$$
\gamma_{\tilde{\Phi}}^{+}(\theta)=(1-\tilde{\zeta} \tilde{\lambda}(\theta)) \gamma_{\bar{\Phi}}^{+}(\theta)+\tilde{\zeta} \tilde{\lambda}(\theta) \gamma_{\bar{\Phi}}^{-}(\theta) \geq(1-\tilde{\zeta}) \gamma_{\bar{\Phi}}^{+}(\theta)>0
$$

In the same way, we get $\gamma_{\tilde{\Phi}}^{-}(\theta)>0$ for $\theta \in \tilde{J}^{\prime} \backslash \bigcup_{i=0}^{q-1} J^{\prime}+i \omega$. Hence, we have that (4.5) holds for all $\theta \in \tilde{J}^{\prime}$. By Lemma 4.3 however, this means that $\gamma_{\tilde{\Phi}}^{ \pm}(\theta)>0$ for all $\theta \in \mathbb{T}^{1}$, and thus $\pi_{1}\left(B^{+}(\tilde{\varphi})\right)=\pi_{1}\left(B^{-}(\tilde{\varphi})\right)=\mathbb{T}^{1}$. Moreover, as ${\underset{\mathcal{C}}{\mathcal{C}^{0}}}_{(}\left(\psi_{3}, \bar{\varphi}^{q}\right) \leq 2 C^{\prime} \tilde{\zeta}$, where $C^{\prime}=\sup _{\theta \in \mathbb{T}^{1}}\left\{\gamma_{\bar{\Phi}}^{+}(\theta), \gamma_{\bar{\Phi}}^{-}(\theta)\right\}<\infty$, Lemma 4.4 implies that if $\tilde{\zeta}$ is small enough, then we have $d_{\mathcal{C}^{0}}(\tilde{\varphi}, \bar{\varphi})<\varepsilon / 4$. This completes the proof.

As mentioned before, even if $f=(\omega, \varphi) \in \mathcal{F}$ satisfies the assertions of Proposition 4.2 and all the fibre maps $f_{\theta}^{q}$ are mode-locked, this does not imply the existence of some topological annulus $\mathcal{A}$ that is mapped inside itself by $f^{q}$. The reason is that possibly neither of $B^{+}(\varphi)$ or $B^{-}(\varphi)$ is essential (meaning that $B(\varphi)$ is doubly essential). As explained in the preceeding section, this is an obstruction to the existence of an annulus $\mathcal{A}$ that is mapped strictly inside itself by some iterate. Hence, we will perturb $\varphi$ to ensure that both $B^{+}$and $B^{-}$consist of finitely many connected components and the distance between each two components of $B^{+}$(resp. $B^{-}$) is positive. This implies immediately that at least one of the two sets is essential. The existence of such a perturbation is established in the following proposition, which corresponds to Step 3. In order to state it, we need to introduce some further notions.

Suppose that $\tilde{B}:=B(\tilde{\varphi})=\left\{(\theta, x) \in \mathbb{T}^{2} \mid \tilde{\Phi}^{q}(\theta, x)=0\right\}$ consists of a finite number of line segments. Call the endpoints of these line segments vertices and further assume that each vertex is the endpoint of exactly two line segments. Then the connected components of $\tilde{B}$ are compact one-dimensional topological manifolds, and thus topological circles. We say a vertex $v=(\theta, x)$ is critical, if there exists $\varepsilon>0$ such that $\pi_{1}\left(B_{\varepsilon}(v) \cap \tilde{B}\right)$ is a half-sided interval with $\theta$ as one endpoint. We say $v$ is a left critical vertex if $\theta$ is the left endpoint of that interval, and a right critical vertex if $\theta$ is the right endpoint. Moreover, we use the same notions for vertices of the lift $\mathcal{B}$ of $\tilde{B}$ to $\mathbb{R}^{2}$. Similar to before, we let $\tilde{B}^{+}:=$ $B^{+}(\tilde{\varphi})=\left\{(\theta, x) \in \mathbb{T}^{2} \mid \tilde{\Phi}^{q}(\theta, x)>0\right\}$ and $\tilde{B}^{-}:=B^{-}(\tilde{\varphi})=\left\{(\theta, x) \in \mathbb{T}^{2} \mid \tilde{\Phi}^{q}(\theta, x)<0\right\}$. 
Proposition 4.5. Suppose that $(\omega, \varphi) \in \mathcal{F}$ and there exists a neighborhood $B_{\eta}(\omega, \varphi)$ in $\mathcal{F}$ such that no $f \in B_{\eta}(\omega, \varphi)$ is mode-locked. Then there exists $\tilde{f}=(\tilde{\omega}, \tilde{\varphi}) \in \mathcal{F}$ such that

(i) $(\tilde{\omega}, \tilde{\varphi})$ satisfies the assertions of Proposition 4.2.

(ii) $\tilde{B}$ consists of a finite number of line segments, and each vertex belongs to exactly two different line segments;

(iii) no line segment in $\tilde{B}$ is vertical ${ }^{3}$

(iv) two different connected components of $\tilde{B}^{+}$have positive distance to each other, and the same is true for $\tilde{B}^{-}$;

(v) every fibre $\{\theta\} \times \mathbb{T}^{1}$ contains at most one critical vertex.

Note that due to (ii), the connected components of $\tilde{B}$ are topological circles. As a consequence, connected components of $\tilde{B}^{-}$and $\tilde{B}^{+}$are either topological disks or annuli, or punctured topological disks or annuli.

Proof. Fix $\varepsilon>0$. Without loss of generality, we may assume that $(\omega, \varphi)$ satisfies the assertions of Proposition 4.2 In particular $\omega=p / q$ is rational. We will not change the rotation number in the base and thus set $\tilde{\omega}=\omega$.

In order to find the desired approximation $\tilde{\varphi}$, we work on the level of lifts and construct an approximation $\tilde{\Phi}$ of $\Phi$, where $\Phi: \mathbb{T}^{2} \rightarrow \mathbb{R}$ is a lift of $\varphi$. To that end, we first fix a triangularisation $\Delta=\left\{D_{i} \mid i=1, \ldots, m\right\}$ of $\mathbb{T}^{2}$ such that

$$
\operatorname{diam}\left(D_{i}\right)<\frac{1}{4} \min _{k=1}^{q-1} d(0, k \omega) .
$$

Further, we require that all the vertices of the triangles in $\Delta$ and their first $q-1$ iterates lie in pairwise different fibres. Note that the latter only depends on the projections of the vertices to the $\theta$-coordinate and on the rotation number $\omega$, but not on the fibre dynamics. Denote by $v_{i}^{j}$ with $j=1,2,3$ the three vertices of $D_{i}$. Obviously, we may have $v_{i}^{j}=v_{i^{\prime}}^{j^{\prime}}$ for $(i, j) \neq\left(i^{\prime}, j^{\prime}\right)$, but every vertex belongs only to a finite number of triangles and the diameter of the union of all triangles containing a given vertex is smaller than $\min _{k=1}^{q-1} d(0, k \omega) / 2$.

Now, we first approximate $\Phi$ by a continuous map $\tilde{\Phi}$ which is piecewise affine on every triangle $D_{i} \in \Delta$. This means that, $\tilde{\Phi}$ is uniquely determined by the values $a_{i}^{j}=\tilde{\Phi}\left(v_{i}^{j}\right)$ (where $a_{i}^{j}=a_{i^{\prime}}^{j^{\prime}}$ whenever $v_{i}^{j}=v_{i^{\prime}}^{j^{\prime}}$ ). Moreover, if $\sup _{i=1}^{m} \operatorname{diam}\left(D_{i}\right)$ is sufficiently small and $d\left(a_{i}^{j}, \Phi\left(v_{i}^{j}\right)\right)<\delta$ for all $i=1, \ldots, m, j=1,2,3$ and some sufficiently small $\delta$, then by uniform continuity $\tilde{\Phi}$ will be $\varepsilon$-close to $\Phi$. In all of the following, we assume that this is the case, which is to say that we choose the triangularisation sufficiently fine and pick all of the $a_{i}^{j}$ from $B_{\delta}\left(\Phi\left(v_{i}^{j}\right)\right)$.

Let $\tilde{\varphi}=\pi \circ \tilde{\Phi}, \tilde{f}=(\omega, \tilde{\varphi})$ and let further

$$
\mathcal{P}_{\tilde{\varphi}}=\bigvee_{n=0}^{q-1} \tilde{f}^{-n}(\Delta)
$$

be the common refinement of the partitions $\tilde{f}^{-n}(\Delta)$ (slightly abusing standard terminology, we allow intersections between the partition elements on their boundaries). Then, since $\tilde{\Phi}$ is piecewise affine, $\mathcal{P}_{\tilde{\varphi}}$ consists of polygons and $\# \mathcal{P}_{\tilde{\varphi}}$ is a finite number that is bounded by some number only depending on $m$ and $q$. Denote by $\operatorname{Vert}\left(\mathcal{P}_{\tilde{\varphi}}\right)$ the set of vertices of polygons in $\mathcal{P}_{\tilde{\varphi}}$. We now show how the $a_{i}^{j}$ can be adjusted to ensure (ii) and (iii), whereas (iv) follows by construction and (v) can be achieved by some extra modification.

(ii) We claim that the $a_{i}^{j}$ can be chosen such that

$$
\tilde{\Phi}^{q}(v) \neq 0 \quad \text { for all } v \in \operatorname{Vert}\left(\mathcal{P}_{\tilde{\varphi}}\right) .
$$

If this holds, then for all $P \in \mathcal{P}_{\tilde{\varphi}}$ the graph of $\tilde{\Phi}_{\mid P}^{q}$, which is the restriction of a hyperplane to the region above $P$, cannot be contained in $\mathbb{R}^{2} \times\{0\}$. Moreover, the intersection of the hyperplane with $\mathbb{R}^{2} \times\{0\}$, which is either empty or a straight line, cannot contain any face or vertex of the polygon. Hence, the intersection

\footnotetext{
${ }^{3}$ Here we say a line segment on the torus is vertical if it lies on some fibre $\{\theta\} \times \mathbb{T}^{1}$
} 
of the graph of $\tilde{\Phi}_{\mid P}^{q}$ with $\mathbb{R}^{2} \times\{0\}$, which projects to $\tilde{B} \cap P$, consists of at most finitely many line segments (note that $P$ may not be convex, so that multiple line segments are possible). Further, the relative interiors of these line segments are contained in the interior of $P$, and their endpoints are contained in the relative interior of two faces of $P$. Since each of these faces belong to exactly one other polygon, each endpoint of a line segments in $\tilde{B} \cap P$ is contained in exactly one further line segment in $\tilde{B}$ that lies in the adjacent polygon.

Since the total number of vertices in $\operatorname{Vert}\left(\mathcal{P}_{\tilde{\varphi}}\right)$ is bounded by some $N \in \mathbb{N}$ depending only on $m$ and $q$, condition (4.7) can be ensured by a successive application of the following claim.

Claim 4.6. Let $M\left(\mathcal{P}_{\tilde{\varphi}}\right)=\left\{v \in \operatorname{Vert}\left(\mathcal{P}_{\tilde{\varphi}}\right) \mid \tilde{\Phi}^{q}(v) \neq 0\right\}$. If $\# M\left(\mathcal{P}_{\tilde{\varphi}}\right)=\ell$ and $\operatorname{Vert}\left(\mathcal{P}_{\tilde{\varphi}}\right) \backslash M\left(\mathcal{P}_{\tilde{\varphi}}\right)$ is non-empty, then an arbitrary small modification of one of the values $a_{i}^{j}$ allows to obtain a perturbation $\bar{\varphi}$ of the same form as $\tilde{\varphi}$ that satisfies $\# M\left(\mathcal{P}_{\bar{\varphi}}\right) \geq \ell+1$.

Proof of the claim. We first note that if $\bar{\varphi}$ is obtained from $\tilde{\varphi}$ by a sufficiently small modification of one of the $a_{i}^{j}$, then for all vertices $v \in M\left(\mathcal{P}_{\tilde{\varphi}}\right)$ there will be a vertex $\bar{v} \in M\left(\mathcal{P}_{\bar{\varphi}}\right)$ arbitrarily close to $v$ that still satisfies $\bar{\Phi}^{q}(\bar{v}) \neq 0$. Thus, $\# M\left(\mathcal{P}_{\bar{\varphi}}\right) \geq \# M\left(\mathcal{P}_{\tilde{\varphi}}\right)$ and it suffices to show that if $\operatorname{Vert}\left(\mathcal{P}_{\tilde{\varphi}}\right) \backslash M\left(\mathcal{P}_{\tilde{\varphi}}\right)$ is nonempty, we may choose $v^{*} \in \operatorname{Vert}\left(\mathcal{P}_{\tilde{\varphi}}\right) \backslash M\left(\mathcal{P}_{\tilde{\varphi}}\right)$ and a perturbation $\bar{\varphi}$ obtained from $\tilde{\varphi}$ by an arbitrarily small modification of one of the $a_{i}^{j}$ such that $v^{*}$ is also contained in $M\left(\mathcal{P}_{\bar{\varphi}}\right)$ but $\bar{\Phi}^{q}(v) \neq 0$.

In order to do so, fix $v^{*}=\left(\theta_{*}, x_{*}\right) \in \operatorname{Vert}\left(\mathcal{P}_{\tilde{\varphi}}\right) \backslash M\left(\mathcal{P}_{\tilde{\varphi}}\right)$. Note that by definition of $\mathcal{P}_{\tilde{\varphi}}$ we must have $v^{*} \in \tilde{f}^{-k}\left(\partial D_{r}\right)$ for some $D_{r} \in \Delta$ and $k \in\{0, \ldots, q-1\}$. Let $v^{\prime}=\left(\theta^{\prime}, x^{\prime}\right)=\tilde{f}^{q-1}\left(v^{*}\right) \in D_{i}$ for some $i \in\{1, \ldots, m\}$ and suppose that $\bar{\varphi}$ is obtained from $\tilde{\varphi}$ by modifying $a_{i}^{j}$ at some vertex $v_{i}^{j}$ of $D_{i}$. Then, due to (4.6) and $\omega=p / q$, we do not modify $\bar{\varphi}(\theta, x)$ for any $\theta$ in one of the intervals $B_{\eta}\left(\theta_{*}\right)+n \omega$ with $\eta=\min _{k=1}^{q-1} d(0, k \omega) / 2, n=0, \ldots, q-2$. However, this means that none of the maps $\tilde{f}, \ldots, \tilde{f}^{q-1}$ is modified in a neighbourhood of $v^{*}$, and by definition of $\mathcal{P}_{\tilde{\varphi}}$ and $\mathcal{P}_{\bar{\varphi}}$, respectively, this means that $v^{*} \in \operatorname{Vert}\left(\mathcal{P}_{\bar{\varphi}}\right)$. Moreover, we have that $\bar{\Phi}^{q}\left(v^{*}\right)=\bar{F}_{\theta^{\prime}}\left(x^{\prime}\right)-x_{*}$, since $\bar{f}_{\theta_{*}}^{q-1}\left(x_{*}\right)=\bar{f}_{\theta_{*}+(q-2) \omega} \circ \cdots \circ \bar{f}_{\theta_{*}}\left(x_{*}\right)=\tilde{f}_{\theta_{*}}^{q-1}\left(x_{*}\right)$. Thus, $\bar{\Phi}^{q}\left(v^{*}\right)$ depends monotonically on $\bar{F}_{\theta^{\prime}}\left(x^{\prime}\right)$. The latter depends monotonically on the values $a_{i}^{j}$, and for at least one $j \in\{1,2,3\}$ this dependence is strictly monotone (recall that $\bar{F}$ is affine on $D_{i}$, so that $\bar{F}_{\mid D_{i}}$ is determined by the choice of the $a_{i}^{j}$ ). Hence, it is possible to modify $a_{i}^{j}$ slightly to ensure that $\bar{\Phi}^{q}(v) \neq 0$. This proves the claim.

(iii) In order to exclude the existence of vertical line segments in $\tilde{B}$, it suffices to ensure that $\partial_{x} \tilde{\Phi}_{\mid \text {int }(P)}^{q} \neq 0$ for all $P \in \mathcal{P}_{\tilde{\varphi}}$. Similar as in (ii), this is a consequence of the following

Claim 4.7. Let $V\left(\mathcal{P}_{\tilde{\varphi}}\right)=\left\{P \in \mathcal{P}_{\tilde{\varphi}} \mid \partial_{x} \tilde{\Phi}_{\mid \operatorname{int}(P)}^{q} \neq 0\right\}$. If $\# V\left(\mathcal{P}_{\tilde{\varphi}}\right)=\ell$ and $\mathcal{P}_{\tilde{\varphi}} \backslash V\left(\mathcal{P}_{\tilde{\varphi}}\right)$ is non-empty, then an arbitrary small modification of one of the values $a_{i}^{j}$ allows to obtain a perturbation $\bar{\varphi}$ of the same form as $\tilde{\varphi}$ that satisfies $\# V\left(\mathcal{P}_{\bar{\varphi}}\right) \geq$ $\ell+1$.

Proof of the claim. As in the previous claim, if $\bar{\varphi}$ is sufficiently close to $\tilde{\varphi}$, then the polygons in $V\left(\mathcal{P}_{\tilde{\varphi}}\right)$ will persists (possibly in slightly perturbed form) and still belong to $V\left(\mathcal{P}_{\bar{\varphi}}\right)$. Hence, we choose some $P \in \mathcal{P}_{\tilde{\varphi}} \backslash V\left(\mathcal{P}_{\tilde{\varphi}}\right)$. Then $\tilde{f}^{q-1}(P)$ is contained in some $D_{i} \in \Delta$. As before, if we modify $a_{i}^{j}$ at one of the vertices $v_{i}^{j}$ of $D_{i}$, then we will not alter $\tilde{f}$ on any of the polygons $P, \tilde{f}(P), \ldots, \tilde{f}^{q-2}(P)$ (note that the horizontal extent of these polygons is always the same and smaller than that of $D_{i}$ ). Hence, $P$ remains unchanged, and the slope of $\bar{\Phi}^{q}$ in the $x$-direction on $P$ depends strictly monotonically on at least one of the values $a_{i}^{j}$. This allows to perform the required modification to render $\partial_{x} \bar{\Phi}_{\mid \text {int }(P)}^{q} \neq 0$.

(iv) By construction, whenever we cross a line segment in $\tilde{B}$, the sign of $\tilde{\Phi}^{q}$ changes. Hence, two different connected components of $\tilde{B}^{-}$, respectively $\tilde{B}^{+}$, cannot lie directly next to each other, so there is a minimal distance between two connected components of the same set. 
(v) Finally, if there are two critical vertices in $\operatorname{Vert}(\tilde{B})$ on the same fibre $\{\theta\} \times \mathbb{T}^{1}$, then we can use Lemmas 4.3 and 4.4 again to perform a perturbation on $B_{\epsilon}(\theta) \times \mathbb{T}^{1}$, where $\epsilon>0$ is sufficiently small and such that there are no critical vertices of $\tilde{B}$ in $\left(B_{\epsilon}(\theta) \backslash\{\theta\}\right) \times \mathbb{T}^{1}$, that moves these critical vertices to different fibres. Note here that critical vertices are always mapped to critical vertices (which may not be true for arbitrary vertices).

It remains to show that any $(\tilde{\omega}, \tilde{\varphi}) \in \mathcal{F}$ satisfying the assertions of Proposition 4.5 is either mode-locked, or we can find some perturbation of it that is mode-locked. This is the content of the following proposition, which corresponds to Step 4 in Section 3 and completes the proof of Theorem 1.4

Before we turn to the precise statement and proof, we briefly comment on the strategy. We assume that a perturbation $\tilde{f}=(\tilde{\omega}, \tilde{\varphi})$ of our original system satisfies the assertions of Proposition 4.5. Then, in the simplest case, both sets $\tilde{B}^{-}$and $\tilde{B}^{+}$may be essential and contain two homotopically non-trivial closed curves $\gamma^{-}$and $\gamma^{+}$, respectively. In this situation, $\gamma^{-}$is disjoint from its image and mapped in the clockwise direction by $\tilde{f}^{q}$, whereas $\gamma^{+}$is mapped in the counterclockwise direction. As a consequence, the annulus which is bounded below by $\gamma^{+}$and above by $\gamma^{-}$is mapped strictly inside itself, so that $\tilde{f}$ is mode-locked. The main problem in the general case is that one of the two sets $\tilde{B}^{ \pm}$, say $\tilde{B}^{-}$, may not be essential. In this case, the boundary of any homotopically non-trivial annulus $\mathcal{A}$ has to intersect $\tilde{B}$. As points in $\tilde{B}$ are fixed by $\tilde{f}^{q}$, this means $\mathcal{A}$ cannot be mapped strictly inside itself.

The idea is now to construct a homotopically non-trivial closed curve $\gamma^{-}$which contains some vertical segments, and while $\gamma^{-}$is allowed to intersect $\tilde{B}$ and also $\tilde{B}^{+}$, this happens only inside these segments. Hence, all the non-vertical parts of $\gamma^{-}$will be contained in $\tilde{B}^{-}$and therefore be mapped in the clockwise direction. Then the vertical segments will still intersect their images, but they can be rendered disjoint from their images by slightly changing the rotation number $\tilde{\omega}$ in the base. If the vertical and nonvertical parts of $\gamma^{-}$are then arranged in the right way, one can ensure that the whole curve $\gamma^{-}$is disjoint from its image and mapped in the clockwise direction. This will then also remain true for a small modification of $\gamma^{-}$in which the vertical segments are slightly tilted, so that locally $\gamma^{-}$can be represented as the graph of a function over the base $\mathbb{T}^{1}$. The construction of $\gamma^{+}$works in an analoguous way, and in fact, both curves have to be constructed simultaneously in order to ensure that the homotopy types coincide.

Proposition 4.8. Let $\tilde{f}=(\tilde{\omega}, \tilde{\varphi}) \in \mathcal{F}$ satisfy the assertions of Proposition 4.5 . Then either $\tilde{f}$ is mode-locked, or there exists $s>0$ such that for any other rotation number $\omega^{\prime} \in(\tilde{\omega}, \tilde{\omega}+s)$ the map $\left(\omega^{\prime}, \tilde{\varphi}\right) \in \mathcal{F}$ is mode-locked.

Before we turn to the proof, we first need to introduce some further notation. Let $\mathcal{B}, \mathcal{B}^{ \pm}$be the lifts of $\tilde{B}, \tilde{B}^{ \pm}$in $\mathbb{R}^{2}$. We denote by VP the set of vertices of $\mathcal{B}$, and by $\mathrm{CV}$ and RCV the sets of critical vertices and right critical vertices, respectively. For any triple $(\hat{\theta}, \hat{x}, \hat{y}) \in \mathbb{R}^{3}$, we say $(\hat{\theta}, \hat{y})$ is in the shadow of $(\hat{\theta}, \hat{x})$ (or $(\hat{\theta}, \hat{x})$ shadows $(\hat{\theta}, \hat{y})$ ) if $\hat{y}<\hat{x},(\hat{\theta}, \hat{x}) \in \mathcal{B}^{-},(\hat{\theta}, \hat{y}) \in \mathcal{B}^{+}$, and there is exactly one $\hat{\xi} \in(\hat{y}, \hat{x}) \subseteq \mathbb{R}$ with $(\hat{\theta}, \hat{\xi}) \in \mathcal{B}$. Then, by the assertions of Proposition 4.5, if $\left(\{\hat{\theta}\} \times \mathbb{R}^{1}\right) \cap C V=\emptyset$ and $(\hat{\theta}, \hat{x}) \in \mathcal{B}^{-}$there always exists some $(\hat{\theta}, \hat{y}) \in \mathcal{B}^{+}$in the shadow of $(\hat{\theta}, \hat{x})$. We define

$$
\mathcal{S}=\left\{(\hat{\theta}, \hat{x}, \hat{y}) \in \mathbb{R}^{3} \mid(\hat{\theta}, \hat{y}) \text { is in the shadow of }(\hat{\theta}, \hat{x})\right\} .
$$

For $(\hat{\theta}, \hat{x}, \hat{y}) \in \mathcal{S}$ and $\hat{\theta}^{\prime} \geq \hat{\theta}$, let

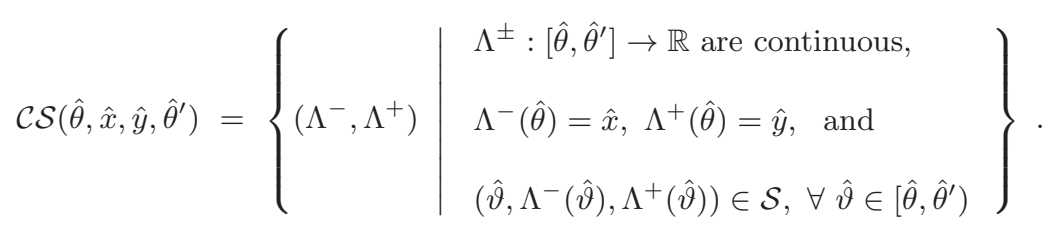

Further, we let

$$
\hat{\theta}^{*}(\hat{\theta}, \hat{x}, \hat{y})=\sup \left\{\hat{\theta}^{\prime} \in \mathbb{R} \mid \exists\left(\Lambda^{-}, \Lambda^{+}\right) \in \mathcal{C S}\left(\hat{\theta}, \hat{x}, \hat{y}, \hat{\theta}^{\prime}\right)\right\} .
$$


Obviously $\hat{\theta}^{*}(\hat{\theta}, \hat{x}, \hat{y}) \geq \hat{\theta}$. Moreover, the following statement holds.

Lemma 4.9. Let $(\hat{\theta}, \hat{x}, \hat{y}) \in \mathcal{S}$. Then $\hat{\theta}^{*}=\hat{\theta}^{*}(\hat{\theta}, \hat{x}, \hat{y})>\hat{\theta}$. Furthermore,

- if $\hat{\theta}^{*}=\infty$, then there exist closed curves $\gamma^{ \pm} \subseteq \tilde{B}^{ \pm}$and they are in the same homotopy class. Moreover, the lifts $\Gamma^{ \pm}$of $\gamma^{ \pm}$in $\mathbb{R}^{2}$ are both simple curves;

- otherwise, the $\operatorname{set} \mathcal{C S}\left(\hat{\theta}, \hat{x}, \hat{y}, \hat{\theta}^{*}\right)$ is non-empty and there exists $\hat{x}^{*} \in \mathbb{R}$ with $\left(\hat{\theta}^{*}, \hat{x}^{*}\right) \in$ $R C V$ such that for any $\left(\Lambda^{-}, \Lambda^{+}\right) \in \mathcal{C S}\left(\hat{\theta}, \hat{x}, \hat{y}, \hat{\theta}^{*}\right)$ either $\Lambda^{-}\left(\hat{\theta}^{*}\right)=\hat{x}^{*}$ or $\Lambda^{+}\left(\hat{\theta}^{*}\right)=$ $\hat{x}^{*}$.

Proof. Let $\theta=\pi(\hat{\theta})$ and $\tilde{B}_{\theta}^{-}=\left(\{\theta\} \times \mathbb{T}^{1}\right) \cap \tilde{B}^{-}=\cup_{i=1}^{N_{\theta}} J_{\theta}^{i}$ where $J_{\theta}^{i}$ are the connected components of $\tilde{B}_{\theta}^{-}$. As $\tilde{B}$ consists only of a finite number of line segments and $\partial \tilde{B}_{\theta}^{-} \subseteq \tilde{B}$, we have $N_{\theta}<\infty$. If $\hat{\theta}^{*}=\infty$, then there exist simple curves $\left(\Lambda^{-}, \Lambda^{+}\right) \in \mathcal{C} \mathcal{S}\left(\hat{\theta}, \hat{x}, \hat{y}, \hat{\theta}+N_{\theta}\right)$. Hence, there exist $i, j \in\left\{1, \ldots, N_{\theta}\right\}$ such that $\pi\left(\Lambda^{-}(\hat{\theta}+i)\right)$ and $\pi\left(\Lambda^{-}(\hat{\theta}+j)\right)$ belong to the same component of $\tilde{B}_{\theta}^{-}$. Without loss of generality we assume $i=0$. Then we can find some $k \in \mathbb{Z}$ such that $\hat{x}+k$ and $\Lambda^{-}(\hat{\theta}+j)$ belong to the same component of $\mathcal{B}^{-}$. Since $\mathcal{B}^{-}$is open and contains the whole vertical line segment between these two points, it is possible to modify $\Lambda^{-}$in a neighbourhood of $\hat{\theta}+j$ in order to obtain $\Lambda^{-}(\hat{\theta}+j)=\hat{x}+k$ without leaving $\mathcal{B}^{-}$. Moreover, by the definition of shadow points, we know that at the same time $\Lambda^{+}(\hat{\theta}+j)$ and $\hat{y}+k$ belong to the same component of $\mathcal{B}^{+} \cap\{\hat{\theta}+j\} \times \mathbb{R}$, and we can modify $\Lambda^{+}$such that $\Lambda^{+}(\hat{\theta}+j)=\hat{y}+k$ without leaving $\mathcal{B}^{+}$. Then the projections $\gamma^{ \pm}$of $\Lambda^{ \pm}$in $\mathbb{T}^{2}$ are closed curves in the same homotopy class with $\gamma^{ \pm} \subseteq \tilde{B}^{ \pm}$as required.

Now we consider the case when $\hat{\theta}^{*}<\infty$ and let $\theta^{*}=\pi\left(\hat{\theta}^{*}\right)$. Further, we define $\mathrm{CV}_{\hat{\theta}^{*}}=\left(\left\{\hat{\theta}^{*}\right\} \times \mathbb{R}\right) \cap \mathrm{CV}$ and $\mathrm{RCV}_{\hat{\theta}^{*}}=\left(\left\{\hat{\theta}^{*}\right\} \times \mathbb{R}\right) \cap \mathrm{RCV}$ and denote by $\mathrm{LS}_{\hat{\theta}^{*}}$ the set of line segments $\sigma$ of $\mathcal{B}$ with $\hat{\theta}^{*} \in \pi_{1}(\sigma)$. Due to $\# \pi(\mathrm{VP})<\infty$, there exists $\eta_{0}>0$ such that

$$
\left(\left(B_{\eta_{0}}\left(\hat{\theta}^{*}\right) \backslash\left\{\hat{\theta}^{*}\right\}\right) \times \mathbb{R}^{1}\right) \cap \mathrm{VP}=\emptyset
$$

Then for any $\sigma \in \operatorname{LS}_{\hat{\theta}^{*}}$, each endpoint of $\sigma$ either belongs to $\left\{\hat{\theta}^{*}\right\} \times \mathbb{R}$ or is outside $B_{\eta_{0}}\left(\hat{\theta}^{*}\right) \times \mathbb{R}$. Due to choice of $\hat{y}$ in the shadow of $\hat{x}$, this makes it easy to see that $\hat{\theta}^{*}>\hat{\theta}$.

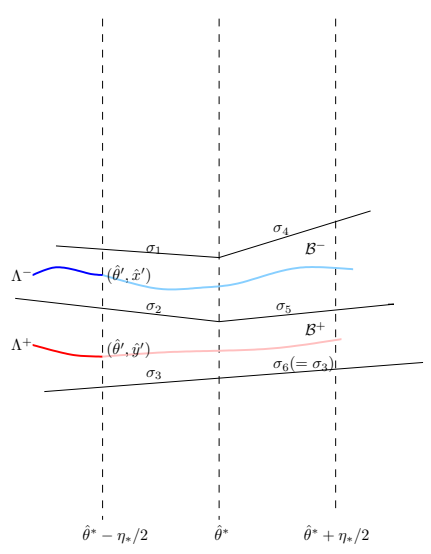

(a)

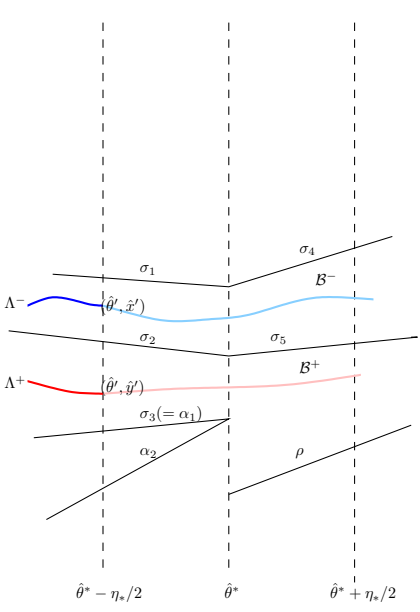

(b)

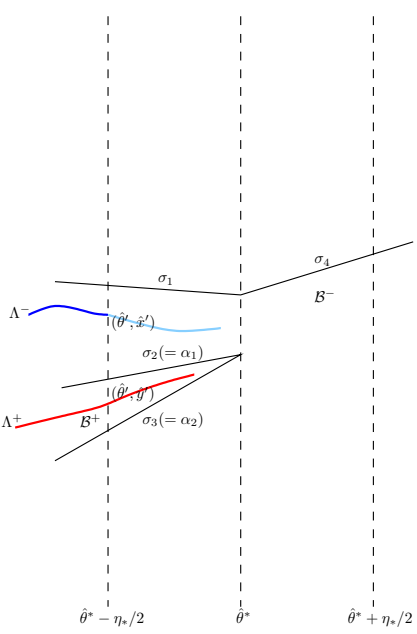

(c)

Figure 4.1: Possible configurations for the line segments in $\operatorname{LS}_{\hat{\theta}^{*}}$.

Now, let $\eta_{*}=\min \left\{\hat{\theta}^{*}-\hat{\theta}, \eta_{0}\right\}>0$. Then by the definition of $\hat{\theta}^{*}$ there exist continuous curves $\Lambda^{ \pm}:\left[\hat{\theta}, \hat{\theta}^{*}-\eta_{*} / 2\right] \rightarrow \mathbb{R}$ with $\Lambda^{-}(\hat{\theta})=\hat{x}, \Lambda^{+}(\hat{\theta})=\hat{y}$ and

$$
\left(\hat{\vartheta}, \Lambda^{-}(\hat{\vartheta}), \Lambda^{+}(\hat{\vartheta})\right) \in \mathcal{S} \quad \forall \hat{\vartheta} \in\left[\hat{\theta}, \hat{\theta}^{*}-\eta_{*} / 2\right] .
$$


Let $\left(\hat{\theta}^{\prime}, \hat{x}^{\prime}, \hat{y}^{\prime}\right):=\left(\hat{\theta}^{*}-\eta_{*} / 2, \Lambda^{-}\left(\hat{\theta}^{*}-\eta_{*} / 2\right), \Lambda^{+}\left(\hat{\theta}^{*}-\eta_{*} / 2\right)\right) \in \mathcal{S}$ and $\theta^{\prime}=\pi\left(\hat{\theta}^{\prime}\right)$. Then, due to (4.8), there exist $\sigma_{1}, \sigma_{2}, \sigma_{3} \in \operatorname{LS}_{\hat{\theta}^{*}}$ such that $\sigma_{3}\left(\hat{\theta}^{\prime}\right)<\hat{y}^{\prime}<\sigma_{2}\left(\hat{\theta}^{\prime}\right)<\hat{x}^{\prime}<\sigma_{1}\left(\hat{\theta}^{\prime}\right)$ and except $\sigma_{2}$ there is no other line segment of $\mathcal{B}$ between $\sigma_{3}$ and $\sigma_{1}$ over the interval $\left(\hat{\theta}^{*}-\eta_{*}, \hat{\theta}^{*}\right)$.

Now, we show that $\mathrm{CV}_{\hat{\theta}^{*}} \neq \emptyset$ if $\hat{\theta}^{*}<\infty$. Suppose this is not the case. Then for the above chosen $\sigma_{1}, \sigma_{2}, \sigma_{3} \in L S_{\hat{\theta}^{*}}$, we have $\sigma_{1}\left(\hat{\theta}^{*}\right) \neq \sigma_{2}\left(\hat{\theta}^{*}\right) \neq \sigma_{3}\left(\hat{\theta}^{*}\right)$. Otherwise, the point $\left(\hat{\theta}^{*}, \sigma_{2}\left(\hat{\theta}^{*}\right)\right)$ is a critical vertex, which is a contradiction to $\mathrm{CV}_{\hat{\theta}^{*}}=\emptyset$. Then for $\iota=1,2,3$, if $\hat{\theta}^{*} \in \operatorname{int}\left(\pi_{1}\left(\sigma_{\iota}\right)\right)$, we let $\sigma_{\iota+3}=\sigma_{\iota}$. Otherwise, there exists another $\beta_{\iota} \in \operatorname{LS}_{\hat{\theta}^{*}}$ with $\beta_{\iota}\left(\hat{\theta}^{*}\right)=\sigma_{\iota}\left(\hat{\theta}^{*}\right)$ and such that $\hat{\theta}^{*}$ is the left endpoint of $\pi_{1}\left(\beta_{\iota}\right)$, and in this case we let $\sigma_{\iota+3}=\beta_{\iota}$. Then by (4.8), except for $\sigma_{5}$ there is no other line segment of $\mathcal{B}$ between $\sigma_{6}$ and $\sigma_{4}$ over the interval $\left(\hat{\theta}^{*}, \hat{\theta}^{*}+\eta_{*}\right)$. Therefore, we can extend $\Lambda^{ \pm}$continuously for $\hat{\vartheta} \in\left[\hat{\theta}^{*}-\eta_{*} / 2, \hat{\theta}^{*}+\eta_{*} / 2\right]$ with $\left(\Lambda^{-}, \Lambda^{+}\right) \in \mathcal{C} \mathcal{S}\left(\hat{\theta}, \hat{x}, \hat{y}, \hat{\theta}^{*}+\eta_{*} / 2\right)$ (see Figure 4.1 (a)). This contradicts the definition of $\hat{\theta}^{*}$.

Hence, we have $\mathrm{CV}_{\hat{\theta}^{*}} \neq \emptyset$. By Proposition 4.5 there exists exactly one critical vertex of $\tilde{B}$ on the fibre $\left\{\theta^{*}\right\} \times \mathbb{T}^{1}$, so there is exactly one critical vertex $\left(\hat{\theta}^{*}, \hat{x}^{*}\right)$ of $\mathcal{B}$ in the line segment $\left\{\hat{\theta}^{*}\right\} \times\left[\sigma_{3}\left(\hat{\theta}^{*}\right), \sigma_{3}\left(\hat{\theta}^{*}\right)+1\right)$. We denote by $\alpha_{1}$ and $\alpha_{2}$ the two line segments of $\mathcal{B}$ containing $\left(\hat{\theta}^{*}, \hat{x}^{*}\right)$. If $\hat{x}^{*} \notin\left\{\sigma_{\iota}\left(\hat{\theta}^{*}\right) \mid \iota=1,2,3\right\}$, then we can extend $\Lambda^{ \pm}$continuously on the interval $\left[\hat{\theta}^{*}-\eta_{*} / 2, \hat{\theta}^{*}+\eta_{*} / 2\right]$ in the same way as in the situation with $\mathrm{CV}_{\hat{\theta}^{*}}=\emptyset$, which is a contradiction. Therefore, we have $\hat{x}^{*} \in\left\{\sigma_{\iota}\left(\hat{\theta}^{*}\right) \mid \iota=1,2,3\right\}$ and $\left(\hat{\theta}^{*}, \hat{x}^{*}\right) \in R C V_{\hat{\theta}^{*}}$ (note that $\left(\hat{\theta}^{*}, \hat{x}^{*}\right)$ cannot be a left critical vertex, since the line segments $\sigma_{\iota}$ with $\iota=1,2,3$ extend to the left of $\left.\hat{\theta}^{*}\right)$. Furthermore, if $\hat{x}^{*} \neq \sigma_{2}\left(\hat{\theta}^{*}\right)$, then $\sigma_{2} \notin\left\{\alpha_{1}, \alpha_{2}\right\}$ and $\sigma_{1}\left(\hat{\theta}^{*}\right) \neq$ $\sigma_{2}\left(\hat{\theta}^{*}\right) \neq \sigma_{3}\left(\hat{\theta}^{*}\right)$. Moreover, either $\hat{x}^{*}=\sigma_{3}\left(\hat{\theta}^{*}\right)$ or $\hat{x}^{*}=\sigma_{1}\left(\hat{\theta}^{*}\right)$. For the case $\hat{x}^{*}=\sigma_{3}\left(\hat{\theta}^{*}\right)$, let $\sigma_{4}, \sigma_{5}$ be as before. Since $\left(\hat{\theta}^{*}, \hat{x}^{*}\right) \in \mathrm{RCV}_{\hat{\theta}^{*}}$, there is $\rho \in \operatorname{LS}_{\hat{\theta}^{*}}$ with $\rho\left(\hat{\theta}^{*}\right)<\sigma_{3}\left(\hat{\theta}^{*}\right)$, and except $\sigma_{5}$ there is no other line segment of $\mathcal{B}$ between $\rho$ and $\sigma_{4}$ over the interval $\left(\hat{\theta}^{*}, \hat{\theta}^{*}+\eta_{*}\right)$ (see Figure 4.1 (b)). Thus, it is easy to see that we can extend $\Lambda^{ \pm}$continuously to the interval $\left[\hat{\theta}^{*}-\eta_{*} / 2, \hat{\theta}^{*}+\eta_{*} / 2\right]$ such that $\left(\Lambda^{-}, \Lambda^{+}\right) \in \mathcal{C S}\left(\hat{\theta}, \hat{x}, \hat{y}, \hat{\theta}^{*}+\eta_{*} / 2\right)$, contradicting the definition of $\hat{\theta}^{*}$. The case where $\hat{x}^{*}=\sigma_{1}\left(\hat{\theta}^{*}\right)$ can be treated similarly.

Therefore, we can assume that $\hat{x}^{*}=\sigma_{2}\left(\hat{\theta}^{*}\right)$ and $\sigma_{2} \in\left\{\alpha_{1}, \alpha_{2}\right\}$. Without loss of generality, we assume that $\alpha_{2}(\hat{\vartheta})<\alpha_{1}(\hat{\vartheta})$ for $\hat{\vartheta} \in\left(\hat{\theta}^{*}-\eta_{*}, \hat{\theta}^{*}\right)$. Now we distinguish two cases.

If $\sigma_{2}=\alpha_{1}$, then by the selection of $\sigma_{1}, \sigma_{2}, \sigma_{3}$, we have $\sigma_{3}=\alpha_{2}$ because there is no other line segment of $\mathcal{B}$ between $\alpha_{2}$ and $\alpha_{1}$ over $\left(\hat{\theta}^{*}-\eta_{*}, \hat{\theta}^{*}\right)$. By the definition of shadowing, for any $\left(\Lambda^{-}, \Lambda^{+}\right) \in \mathcal{C S}\left(\hat{\theta}, \hat{x}, \hat{y}, \hat{\theta}^{*}\right)$ we have that $\alpha_{2}(\hat{\vartheta})<\Lambda^{+}(\hat{\vartheta})<\alpha_{1}(\hat{\vartheta})$ for all $\hat{\vartheta} \in\left(\hat{\theta}^{*}-\eta_{*}, \hat{\theta}^{*}\right)$. Together with $\alpha_{1}\left(\hat{\theta}^{*}\right)=\alpha_{2}\left(\hat{\theta}^{*}\right)=\hat{x}^{*}$ (see Figure 4.1 (c)), we obtain

$$
\Lambda^{+}\left(\hat{\theta}^{*}\right)=\lim _{\hat{\vartheta} \rightarrow \hat{\theta}^{*}} \Lambda^{+}(\hat{\vartheta})=\hat{x}^{*}
$$

If $\sigma_{2}=\alpha_{2}$, then we have $\sigma_{1}=\alpha_{1}$ and similar to the above we conclude that

$$
\Lambda^{-}\left(\hat{\theta}^{*}\right)=\lim _{\hat{\vartheta} \rightarrow \hat{\theta}^{*}} \Lambda^{-}(\hat{\vartheta})=\hat{x}^{*}
$$

This completes the proof.

For $(\hat{\theta}, \hat{x}, \hat{y}) \in \mathcal{S}$, if $\hat{\theta}^{*}=\hat{\theta}^{*}(\hat{\theta}, \hat{x}, \hat{y})<\infty$, we say the point $\left(\hat{\theta}^{*}, \hat{x}^{*}\right) \in \mathrm{RCV}$ obtained in Lemma 4.9 is the target point of $(\hat{\theta}, \hat{x}, \hat{y})$. Moreover, we denote by $\ell_{*}$ the maximal slope of a line segment in $\mathcal{B}$ and note that since $\mathcal{B}$ contains no vertical segments we have $\ell_{*}<\infty$. Now we are ready to prove Proposition 4.8

Proof of Proposition 4.8. Recall that by Definition 1.3 in order to prove that a map in $\mathcal{F}$ is mode-locked, we need to find a closed topological annulus $\mathcal{A}$ that is mapped strictly into itself by some iterate of the map.

Suppose $\tilde{f}=(\tilde{\omega}, \tilde{\varphi}) \in \mathcal{F}$ satisfies the assertions of Proposition 4.5 Note that if we have $\hat{\theta}^{*}(\hat{\theta}, \hat{x}, \hat{y})=\infty$ for some $(\hat{\theta}, \hat{x}, \hat{y}) \in \mathcal{S}$, then Lemma 4.9 implies that there exist closed curves $\gamma^{ \pm} \subseteq \tilde{B}^{ \pm}$in the same homotopy class. Let $\mathcal{A}$ be the annulus that is bounded by these curves and lies between $\gamma^{+}$and $\gamma^{-}$in the counterclockwise direction. Then, since the lifts $\Gamma^{+}$and $\Gamma^{-}$, which are the graphs of continuous functions from $\mathbb{R}$ to $\mathbb{R}$, are mapped above, respectively below themselves by the lift of $\tilde{f}^{q}$, the annulus $\mathcal{A}$ is mapped 
strictly inside itself by $\tilde{f}^{q}$. Hence, we only need to address the case where $\hat{\theta}^{*}(\hat{\theta}, \hat{x}, \hat{y})<\infty$ for all $(\hat{\theta}, \hat{x}, \hat{y}) \in \mathcal{S}$.

Due to Proposition 4.5 we have that $\epsilon=\min \left\{d\left(\vartheta^{\prime}, \vartheta^{\prime \prime}\right) \mid\left(\vartheta^{\prime}, \xi^{\prime}\right) \neq\left(\vartheta^{\prime \prime}, \xi^{\prime \prime}\right) \in\right.$ $\pi(\mathrm{CV})\}>0$. Choose $\left(\hat{\theta}, \hat{x}^{-}, \hat{x}^{+}\right) \in \mathcal{S}$ such that for any $(\hat{\vartheta}, \hat{\xi}) \in \mathrm{CV}$ we have $d(\hat{\theta}, \hat{\vartheta}) \geq \epsilon / 2$. We claim that for any $N \in \mathbb{N}$ there exist continuous curves

$$
\Gamma_{N}^{ \pm}=\bigcup_{i=0}^{N} \Lambda_{i}^{ \pm} \cup \bigcup_{i=1}^{N} S_{i}^{ \pm}
$$

such that for all $i=0, \ldots, N$ we have

(i) $\pi_{1}\left(\Lambda_{i}^{+}\right)=\pi_{1}\left(\Lambda_{i}^{-}\right)=\left[\hat{\theta}_{i-1}, \hat{\theta}_{i}\right]$ is a closed interval, denoted by $I_{i}$, with $\Lambda_{i \mid I_{i}}^{ \pm} \subseteq \mathcal{B}^{ \pm}$, $\left|I_{i}\right|>\epsilon / 3, \hat{\theta}_{-1}=\hat{\theta}$ and for $i \geq 0$ there exists $\left(\hat{\theta}_{i}^{*}, \hat{x}_{i}^{*}\right) \in \mathrm{RCV}$ such that $\hat{\theta}_{i}=$ $\hat{\theta}_{i}^{*}-\epsilon / 10$

(ii) $\left(\Lambda_{i}^{-}, \Lambda_{i}^{+}\right) \in \mathcal{C S}\left(\hat{\theta}_{i-1}+\epsilon / 5, \Lambda_{i}^{-}\left(\hat{\theta}_{i-1}+\epsilon / 5\right), \Lambda_{i}^{+}\left(\hat{\theta}_{i-1}+\epsilon / 5\right), \hat{\theta}_{i}\right)$ and $\left(\hat{\theta}_{i}^{*}, \hat{x}_{i}^{*}\right)$ is the target point of $\left(\hat{\theta}_{i}-\epsilon / 10, \Lambda_{i}^{-}\left(\hat{\theta}_{i}-\epsilon / 10\right), \Lambda_{i}^{+}\left(\hat{\theta}_{i}-\epsilon / 10\right)\right) \in \mathcal{S}$

(iii) either $S_{i+1}^{+}=\emptyset, \Lambda_{i}^{+}\left(\hat{\theta}_{i}\right)=\Lambda_{i+1}^{+}\left(\hat{\theta}_{i}\right)$ and $S_{i+1}^{-}$is a vertical segment over $\hat{\theta}_{i}$ connecting $\Lambda_{i}^{-}$and $\Lambda_{i+1}^{-}$with $\Lambda_{i}^{-}\left(\hat{\theta}_{i}\right)<\Lambda_{i+1}^{-}\left(\hat{\theta}_{i}\right)$, or $S_{i+1}^{-}=\emptyset, \Lambda_{i}^{-}\left(\hat{\theta}_{i}\right)=\Lambda_{i+1}^{-}\left(\hat{\theta}_{i}\right)$ and $S_{i+1}^{+}$is a vertical segment over $\hat{\theta}_{i}$ connecting $\Lambda_{i}^{+}$and $\Lambda_{i+1}^{+}$with $\Lambda_{i}^{+}\left(\hat{\theta}_{i}\right)>\Lambda_{i+1}^{+}\left(\hat{\theta}_{i}\right)$.

In order to start the inductive construction, we let $\hat{\theta}_{-1}=\hat{\theta}$ and apply Lemma 4.9 to obtain $\left(\bar{\Lambda}_{0}^{-}, \bar{\Lambda}_{0}^{+}\right) \in \mathcal{C S}\left(\hat{\theta}, \hat{x}^{-}, \hat{x}^{+}, \hat{\theta}_{0}^{*}\right)$, where $\left(\hat{\theta}_{0}^{*}, \hat{x}_{0}^{*}\right)$ is the target point of $\left(\hat{\theta}, \hat{x}^{-}, \hat{x}^{+}\right)$. Then we let $I_{0}=\left[\hat{\theta}, \hat{\theta}_{0}^{*}-\epsilon / 10\right]$ and $\Lambda_{0}^{ \pm}=\bar{\Lambda}_{0 \mid I_{0}}^{ \pm}$. It is easy to check that (i) and (ii) hold, whereas (iii) is still void.

Now, assume that we have already constructed $\Lambda_{i}^{ \pm}$for $i=0,1, \ldots, \iota$ and $S_{i}^{ \pm}$for $i=1, \ldots, \iota$. Since $\hat{\theta}_{\iota}=\hat{\theta}_{\iota}^{*}-\epsilon / 10$ by (i) and due to the choice of $\epsilon$, we know that there is no critical vertex in $\left(\hat{\theta}_{\iota}-\epsilon / 5, \hat{\theta}_{\iota}^{*}\right) \times \mathbb{R}$. Further, $\left(\hat{\theta}_{\iota}^{*}, \hat{x}_{\iota}^{*}\right)$ is the target point of $\left(\hat{\theta}_{\iota}-\epsilon / 10, \Lambda_{\iota}^{-}\left(\hat{\theta}_{\iota}-\epsilon / 10\right), \Lambda_{\iota}^{+}\left(\hat{\theta}_{\iota}-\epsilon / 10\right)\right)$. Hence, by Lemma 4.9 we can continuously extend $\Lambda_{\iota}^{ \pm}$to continuous curves $\bar{\Lambda}_{\iota}^{ \pm}$defined on the larger interval $\left[\hat{\theta}_{\iota-1}, \hat{\theta}_{\iota}^{*}\right]$. (In fact, the $\Lambda_{i}^{ \pm}$are defined as the restrictions of such longer curves in the inductive construction, see below.) There are two possibilities

(1) $\bar{\Lambda}_{\iota}^{-}\left(\hat{\theta}_{\iota}^{*}\right)=\hat{x}_{\iota}^{*}$;

(2) $\bar{\Lambda}_{\iota}^{+}\left(\hat{\theta}_{\iota}^{*}\right)=\hat{x}_{\iota}^{*}$.

Case (1): If $\bar{\Lambda}_{\iota}^{-}\left(\hat{\theta}_{\iota}^{*}\right)=\hat{x}_{\iota}^{*}$, we let $S_{\iota+1}^{+}=\emptyset$ and choose $S_{\iota+1}^{-}$to be a vertical segment with endpoints $\left(\hat{\theta}_{\iota}, a\right),\left(\hat{\theta}_{\iota}, b\right)$ where $a=\Lambda_{\iota}^{-}\left(\hat{\theta}_{\iota}\right)$ and $b>a$ is chosen such that $\left(\hat{\theta}_{\iota}, a\right)$ and $\left(\hat{\theta}_{\iota}, b\right)$ belong to two successive connected components of $\mathcal{B}_{\hat{\theta}_{\iota}}^{-}=\left(\left\{\hat{\theta}_{\iota}\right\} \times \mathbb{R}\right) \cap \mathcal{B}^{-}$. Since there is no critical vertex with first coordinate $\hat{\theta}_{\iota}$, there exists a point $\left(\hat{\theta}_{\iota}, c\right) \in \mathcal{B}^{+}$in the shadow of $\left(\hat{\theta}_{\iota}, b\right)$. Then, by Lemma 4.9 there exists a unique target point $\left(\hat{\theta}_{\iota+1}^{*}, \hat{x}_{\iota+1}^{*}\right)$ of $\left(\hat{\theta}_{\iota}, b, c\right) \in \mathcal{S}$ and there is $\left(\bar{\Lambda}_{\iota+1}^{-}, \bar{\Lambda}_{\iota+1}^{+}\right) \in \mathcal{C S}\left(\hat{\theta}_{\iota}, b, c, \hat{\theta}_{\iota+1}^{*}\right)$. By the choice of $b$, we have that $\hat{\theta}_{\iota+1}^{*} \neq \hat{\theta}_{\iota}^{*}$ and hence $\hat{\theta}_{\iota+1}^{*}>\hat{\theta}_{\iota}^{*}$.

Since there is no other critical vertex in $B_{\epsilon / 2}\left(\theta_{\iota}^{*}\right) \times \mathbb{T}^{1}$, there exists $0<\delta \leq \epsilon / 10$ such that the line segment $\lambda$ connecting the points $\left(\hat{\theta}_{\iota}^{*}, \bar{\Lambda}_{\iota}^{+}\left(\hat{\theta}_{\iota}^{*}\right)\right)$ and $\left(\hat{\theta}_{\iota}^{*}+\delta, \bar{\Lambda}_{\iota+1}^{+}\left(\hat{\theta}_{\iota}^{*}+\delta\right)\right)$ is contained in $\mathcal{B}^{+}$(see Figure 4.2(Case (1))). Now we let $\hat{\theta}_{\iota+1}=\hat{\theta}_{\iota+1}^{*}-\epsilon / 10, I_{\iota+1}=\left[\hat{\theta}_{\iota}, \hat{\theta}_{\iota+1}\right]$ and set $\Lambda_{\iota+1}^{-}=\bar{\Lambda}_{\iota+1 \mid I_{\iota+1}}^{-}$. Further, we define $\Lambda_{\iota+1}^{+}$as

$$
\Lambda_{\iota+1}^{+}=\bar{\Lambda}_{\iota \mid\left[\hat{\theta}_{\iota}, \hat{\theta}_{\iota}^{*}\right]}^{+} \cup \lambda \cup \bar{\Lambda}_{\iota+1 \mid\left[\hat{\theta}_{\iota}^{*}+\delta, \hat{\theta}_{\iota+1}\right]}^{+} .
$$

It is now easy to check that $\Lambda_{\iota+1}^{ \pm}$and $S_{\iota+1}^{ \pm}$satisfy the inductive assumptions (i)-(iii). Moreover, in Case (2) the construction works in completely symmetric way.

Hence, we have that curves $\Gamma_{N}^{ \pm}$with properties (i)-(iii) exist for all $N \in \mathbb{N}$. Now, suppose $M=\# \pi(\mathrm{RCV})$. Then there have to exist $i<j \in\{0, \ldots, M\}$ such that $\pi\left(\hat{\theta}_{i}^{*}, \hat{x}_{i}^{*}\right)=\pi\left(\hat{\theta}_{j}^{*}, \hat{x}_{j}^{*}\right)$ and for any other pair $\left(i^{\prime}, j^{\prime}\right)$ with $i^{\prime} \neq j^{\prime} \in\{i, i+1, \ldots, j\}$, $\pi\left(\hat{\theta}_{i^{\prime}}^{*}, \hat{x}_{i^{\prime}}^{*}\right) \neq \pi\left(\hat{\theta}_{j^{\prime}}^{*}, \hat{x}_{j^{\prime}}^{*}\right)$. Without loss of generality, we assume $i=0$. Then, we have that $\hat{\theta}_{j}^{*}=\hat{\theta}_{0}^{*}+k$ and $\hat{x}_{j}^{*}=\hat{x}_{0}^{*}+m$ for some integers $k, m$, so the target points of $\Lambda_{0}^{ \pm}$ and $\Lambda_{j}^{ \pm}$only differ by the integer vector $(k, m)$. This means that the projections of these 


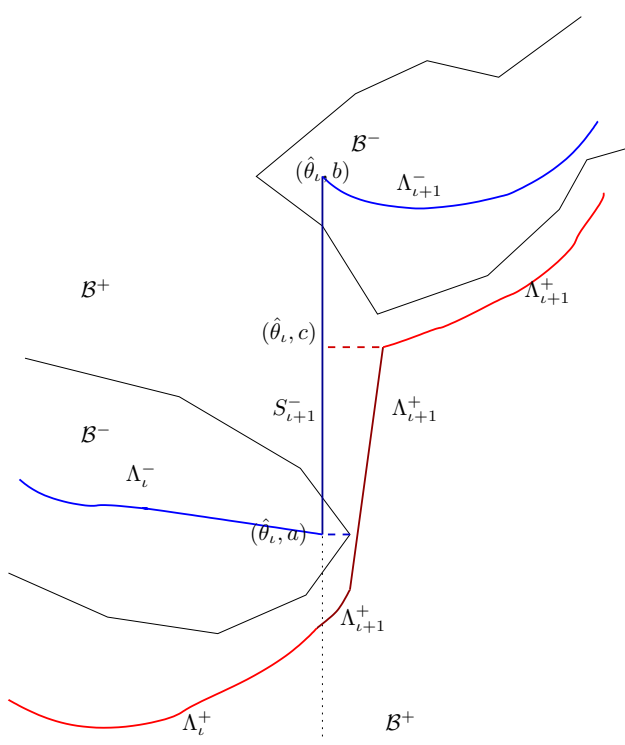

Case (1)

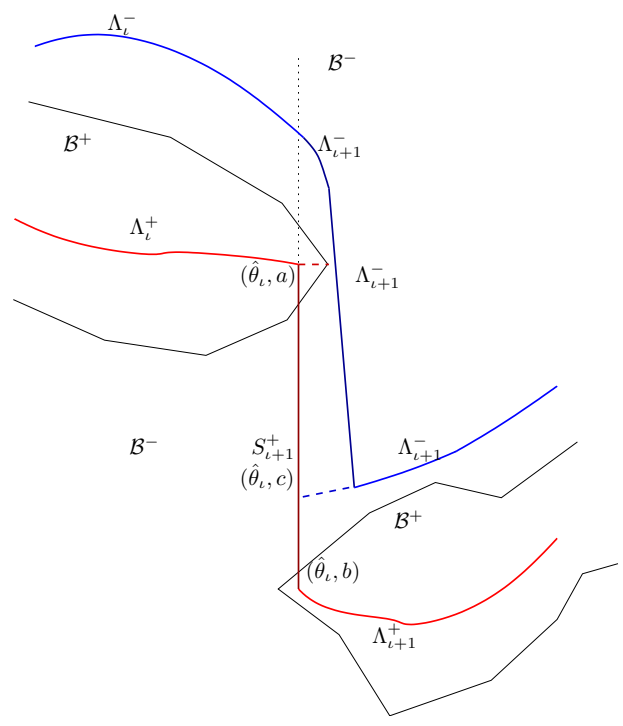

Case (2)

Figure 4.2: The strategy of the construction.

curves to $\mathbb{T}^{2}$ have to be contained in the same connected components of $\tilde{B}^{ \pm}$. Since the sets $\mathcal{B}^{ \pm}$are open, we can thus modify $\Lambda_{j}^{ \pm}$in such a way that for some $\tilde{\delta}>0$ we have $\Lambda_{j}^{ \pm}(\hat{\vartheta}+j)=\Lambda_{0}^{ \pm}(\hat{\vartheta})+k$ for all $\hat{\vartheta} \in\left[\hat{\theta}_{0}-\tilde{\delta}, \hat{\theta}_{0}\right]$. As a consequence, the curves

$$
\bar{\Gamma}^{ \pm}=\bigcup_{i=1}^{N} \Lambda_{i}^{ \pm} \cup \bigcup_{i=1}^{N} S_{i}^{ \pm}
$$

project to closed curves $\gamma^{ \pm}$in the torus. Note that due to the modification of $\Lambda_{j}^{ \pm}$, the projections of these curves end exactly in the starting points of the projection of $\Lambda_{1}^{ \pm}$or $S_{1}^{ \pm}$, respectively. We denote by $\Gamma^{ \pm}$the periodic extensions of the curves $\bar{\Gamma}^{ \pm}$to all of $\mathbb{R}$ that is, the $\Gamma^{ \pm}$are lifts of $\gamma^{ \pm}$to $\mathbb{R}^{2}$.

Finally, we want to see that the existence of the curves $\Gamma^{ \pm}$constructed above implies that for any $\omega^{\prime}$ in a right neighbourhood of $\tilde{\omega}$ the system $\bar{f}=\left(\omega^{\prime}, \tilde{\varphi}\right)$ is mode-locked. This follows if we can show that a suitable lift $\bar{F}^{q}$ of $\bar{f}^{q}$ maps the 'infinite strip'

$$
\widehat{\mathcal{A}}=\left\{(\hat{\vartheta}, \hat{\xi}) \in \mathbb{R}^{2} \mid \Gamma^{+}(\hat{\vartheta}) \leq \hat{\xi} \leq \Gamma^{-}(\hat{\vartheta})\right\}
$$

strictly inside itself. This forces the fibred rotation number of $\bar{f}$ to be equal to $\frac{m}{k q}$, and since this situation is persistent under perturbations of $\tilde{\varphi}$ it implies that $\bar{f}$ is mode-locked (compare 23]).

In order to do so, we choose $\tilde{F}^{q}$ to be a suitable lift of $\tilde{f}^{q}$ to $\mathbb{R}^{2}$ which has rotation number zero in the base, and for $\omega^{\prime}$ slightly to the right of $\tilde{\omega}$ we choose $\bar{F}^{q}$ as the lift of $\bar{f}^{q}$ whose rotation number is close to zero. (Note here that we choose $\tilde{F}^{q}$ directly as a lift of $\tilde{f}^{q}$, and it is not necessarily the iterate of a lift $\tilde{F}$ of $\tilde{f}$, similar to $\bar{F}^{q}$.) Note that for $\tilde{F}^{q}$ itself, the vertical parts of the curves $\Gamma^{ \pm}$contain fixed points (since they intersect both $\mathcal{B}^{-}$and $\mathcal{B}^{+}$), so that $\mathcal{A}$ cannot be mapped strictly inside itself. However, if $\omega^{\prime}$ is slightly larger than $\tilde{\omega}$ then $\bar{F}^{q}$ shifts these vertical segments slightly to the right, so that they become disjoint from their images. It remains to show that the whole curves $\Gamma^{ \pm}$ are also disjoint from their images under $\bar{F}^{q}$. The fact that $\Gamma^{-}$is mapped below and $\Gamma^{+}$is mapped above themselves then follows easily from the fact that this is true for the non-vertical parts of these curves under the map $\tilde{F}^{q}$.

We give the argument for $\Gamma^{-}$, while the proof for $\Gamma^{+}$is analogous. Suppose that $\omega^{\prime} \in(\tilde{\omega}, \tilde{\omega}+s)$, where $s>0$ is small (to be specified below). In order to prove that $\bar{F}^{q}\left(\Gamma^{-}\right) \cap \Gamma^{-}=\emptyset$, we need to show that 
(1) $\bar{F}^{q}\left(\Lambda_{i}^{-}\right) \cap \Lambda_{j}^{-}=\emptyset$ for all $i, j \in\{1, \ldots, N\}$.

(2) $\bar{F}^{q}\left(\Lambda_{i}^{-}\right) \cap S_{j}^{-}=\emptyset$ for all $i, j \in\{1, \ldots, N\}$.

(3) $\bar{F}^{q}\left(S_{j}^{-}\right) \cap \Lambda_{i}^{-}=\emptyset$ for all $i, j \in\{1, \ldots, N\}$.

(1) If $|i-j| \geq 1$, then $\bar{F}^{q}\left(\Lambda_{i}^{-}\right)$and $\Lambda_{j}^{-}$have disjoint projections to the first coordinate, since this is true for $\Lambda_{i}^{-}$and $\Lambda_{j}^{-}$and $\bar{F}^{q}$ only shifts horizontally by $q\left(\omega^{\prime}-\tilde{\omega}\right) \leq q s$, which is arbitrarily small. This is similar for $i=j+1$, since $\bar{F}^{q}$ shifts horizontally to the right.

If $i=j$, then $\tilde{F}^{q}\left(\Lambda_{i}^{-}\right)$and $\Lambda_{i}^{-}$are disjoint by construction $\left(\Lambda_{i}^{-} \subseteq \mathcal{B}^{-}\right)$and therefore have positive distance to each other. Hence, if $s$ is sufficiently small, this remains true if $\tilde{F}^{q}$ is replaced by $\bar{F}^{q}$.

Finally, if $i=j-1$, then the projections of $\bar{F}^{q}\left(\Lambda_{i}^{-}\right)$and $\Lambda_{j}^{-}$have a small interval $\left[\hat{\theta}_{i}, \hat{\theta}_{i}+q\left(\omega^{\prime}-\tilde{\omega}\right)\right] \subseteq\left[\hat{\theta}_{i}, \hat{\theta}_{i}+q s\right]$ of overlap. We have to consider two situations: If $S_{j}^{-}=\emptyset$, then $\Lambda_{i}^{-} \cup \Lambda_{j}^{-}$is a connected curve that is mapped strictly below itself by $\tilde{F}^{q}$. As above, this remains true after perturbed to $\bar{F}^{q}$ if $s$ is sufficiently small. If $S_{j}^{-} \neq \emptyset$, then by construction the endpoints $\left(\hat{\theta}_{i}, \Lambda_{i}^{-}\left(\hat{\theta}_{i}\right)\right)$ and $\left(\hat{\theta}_{i}, \Lambda_{j}^{-}\left(\hat{\theta}_{i}\right)\right)$ of this segment are separated by some interval that belongs to $\mathcal{B}^{+}$. Hence, $\tilde{F}^{q}\left(\hat{\theta}_{i}, \Lambda_{j}^{-}\left(\hat{\theta}_{i}\right)\right)$ lies strictly above $\left(\hat{\theta}_{i}, \Lambda_{i}^{-}\left(\hat{\theta}_{i}\right)\right)$. By continuity, this implies that the overlapping parts of the curves $\bar{F}^{q}\left(\Lambda_{i}^{-}\right)$and $\Lambda_{j}^{-}$are still disjoint if $s$ is sufficiently small.

(2) Similar to before, since $\bar{F}^{q}$ only shifts horizontally by at most $q s$, we only have to consider the cases $i=j-1$ and $i=j$. If $i=j-1$, then $\Lambda_{i}^{-}$connects to $S_{j}^{-}$ in its right endpoint, which is the lower endpoint of $S_{j}^{-}$. As $\Lambda_{i}^{-}$is mapped below itself by $\tilde{F}^{q}$ its image will be disjoint from $S_{j}^{-}$, and the same is still true after perturbation if $s$ is small.

If $i=j$, then the upper endpoint of $S_{j}^{-}$equals the left endpoint of $\Lambda_{i}^{-}$. Since $\bar{F}^{q}$ shifts slightly to the right, this means that the projection $\hat{\theta}_{j-1}$ of $S_{j}^{-}$is not contained in the projection of $\bar{F}^{q}\left(\Lambda_{i}^{-}\right)$, so the two sets are disjoint.

(3) Again, we only have to consider $i=j-1$ and $i=j$. In the first case, $S_{j}^{-}$is mapped to the right by $\bar{F}^{q}$ and therefore has disjoint projection from $\Lambda_{i}^{-}$. In the second case, $S_{j}^{-}$lies below the left endpoint of $\Lambda_{j}^{-}$. Since the upper endpoint of $S_{j}^{-}$is mapped downwards by $\tilde{F}^{q}$, the image $\tilde{F}^{q}\left(S_{j}^{-}\right)$has positive distance to $\Lambda_{i}^{-}$, and as before this remains true after perturbation if $s$ is small.

\section{Proof of Corollary 1.5 and Theorem 1.6}

Corollary 1.5] is a direct consequence of Theorem 1.4 and the following elementary lemma on products of Baire spaces.

Lemma 5.1. Suppose $A, B$ are Baire spaces, $B$ is second-countable, and $U \subseteq A \times B$ is a residual (resp. open and dense) set. Then there exists a residual set $V \subseteq A$, such that for any $v \in V$ there exists a residual (resp. open and dense) set $U_{v} \subseteq B$ such that $\{v\} \times U_{v} \subseteq U$.

In the same way, Theorem 1.6(b) follows from Theorem 1.6(a). Hence, we only need to prove Theorem 1.6 (a).

Proof of Theorem 1.6(a) : We fix an arbitrary dense sequence $\left\{\tau_{n}\right\}_{n \in \mathbb{N}} \subseteq \mathbb{T}^{1}$ and let

$$
\mathcal{P}_{n}=\left\{\left(f_{\tau}\right)_{\tau \in \mathbb{T}^{1}} \in \mathcal{P} \mid f_{\tau_{n}} \text { is mode-locked }\right\} .
$$

If all $\mathcal{P}_{n}$ are open and dense in $\mathcal{P}$, then $\mathcal{P}^{D S}=\cap_{n} \mathcal{P}_{n}$ is residual and satisfies the assertion. Here, it suffices to show that $\mathcal{P}_{n}$ is dense, openness being obvious.

To that end, we fix $\left(f_{\tau}\right)_{\tau \in \mathbb{T}^{1}} \in \mathcal{P}$, and $\varepsilon>0$. Choose $\hat{f} \in B_{\varepsilon / 3}\left(f_{\tau_{n}}\right)$ so that $\hat{f}$ is mode-locked. We write $\hat{f}=(\hat{\omega}, \hat{\varphi}), f_{\tau}=\left(\omega, \varphi_{\tau}\right)$. Then we fix an interval $I=[a, b]$ with $\tau_{n} \in \operatorname{int}(I)$, such that $f_{\tau} \in B_{\varepsilon / 3}\left(f_{\tau_{n}}\right), \forall \tau \in I$. We define

$$
\tilde{f}_{\tau}= \begin{cases}\left(\hat{\omega}, \varphi_{\tau}\right) & \text { if } \tau \notin I, \\ \left(\hat{\omega}, \frac{\tau_{n}-\tau}{\tau_{n}-a} \varphi_{a}+\frac{\tau-a}{\tau_{n}-a} \hat{\varphi}\right) & \text { if } \tau \in\left[a, \tau_{n}\right], \\ \left(\hat{\omega}, \frac{b-\tau}{b-\tau_{n}} \hat{\varphi}+\frac{\tau-\tau_{n}}{b-\tau_{n}} \varphi_{b}\right) & \text { if } \tau \in\left[\tau_{n}, b\right],\end{cases}
$$


where the convex combinations should be interpreted as discussed in Section 2 Then $\left(\tilde{f}_{\tau}\right)_{\tau \in \mathbb{T}^{1}}$ is $\varepsilon$-close to $\left(f_{\tau}\right)_{\tau \in \mathbb{T}^{1}}$ and $\tilde{f}_{\tau_{n}}=\hat{f}$ is mode-locked. This completes the proof.

\section{References}

[1] W. de Melo and S. van Strien. One-dimensional dynamics. Springer, 1993.

[2] A. Katok and B. Hasselblatt. Introduction to the Modern Theory of Dynamical Systems. Cambridge University Press, 1997.

[3] A. Haro and J. Puig. Strange non-chaotic attractors in Harper maps. Chaos, 16, 2006.

[4] J. Béllissard and B. Simon. Cantor spectrum for the almost Mathieu equation. J. Funct. Anal., 48(3):408-419, 1982.

[5] M. Choi, G.A. Elliott and N. Yui, Gauss polynomials and the rotation algebra. Invent. Math. 99(1):225-246, 1990.

[6] J. Puig. Cantor spectrum for the almost Mathieu operator. Commun. Math. Phys., 244(2):297-309, 2004.

[7] A. Avila and S. Jitomirskaya. The Ten Martini Problem. Ann. Math. (2), 170(1):303$342,2009$.

[8] B. Simon. Almost periodic Schrödinger operators: a review. Adv. Math. 3(4): 463-490, 1982.

[9] L.H. Eliasson. Floquet solutions for the 1-dimensional quasi-periodic Schrödinger equation. Commun. Math. Phys., 146:447-482, 1992.

[10] M. Goldstein and W. Schlag. On resonances and the formation of gaps in the spectrum of quasi-periodic Schrödinger equations. Ann. Math. (2), 173(1):337-475, 2011.

[11] A. Avila, J. Bochi and D. Damanik. Cantor spectrum for Schrödinger operators with potentials arising from generalized skew-shifts. Duke Math. J. 146(2):253-280, 2009.

[12] A. Avila, Density of positive Lyapunov exponents for $S L(2, \mathbb{R})$ cocycles. J. Am. Math. Soc. 24:999-1014, 2011.

[13] R. Fabbri, R. Johnson and R.Pavani. On the nature of the spectrum of the quasiperiodic Schrödinger operator. Nonlinear Anal. Real World Appl., 3:37-59, 2002.

[14] M. Benedicks and L. Carleson. The dynamics of the Hénon map. Ann. Math. (2), 133(1):73-169, 1991.

[15] K. Bjerklöv. Positive Lyapunov exponent and minimality for a class of onedimensional quasiperiodic Schrödinger equations. Ergodic Theory Dyn. Syst., $25: 1015-1045,2005$.

[16] T. Jäger. Strange non-chaotic attractors in quasiperiodically forced circle maps. Comm. Math. Phys., 289(1):253-289, 2009.

[17] T. Jäger. Strange non-chaotic attractors in quasiperiodically forced circle maps: Diophantine forcing. Ergodic Theory Dyn. Syst., 2013, 33(5):1477-1501.

[18] Y. Wang and Z. Zhang. Uniform positivity and continuity of Lyapunov exponents for a class of $C^{2}$ quasiperiodic Schrödinger cocycles. J. Funct. Ana., 268(9):2525-2585, 2015 .

[19] Y. Wang and Z. Zhang. Cantor spectrum for a class of $\mathcal{C}^{2}$ quasiperiodic Schrödinger operators. Preprint 2014, arXiv:1410.0101.

[20] T. Jäger and J. Wang. Abundance of mode-locking for quasiperiodically forced circle maps. Preprint 2015, arXiv:1504.03887

[21] R. Johnson and J. Moser. The rotation number for almost periodic potentials. Commun. Math. Phys., 84(3):403-438, 1982.

[22] M. Herman. Une méthode pour minorer les exposants de Lyapunov et quelques exemples montrant le caractère local d'un théorème d'Arnold et de Moser sur le tore de dimension 2. Comment. Math. Helv., 58:453-502, 1983.

[23] K. Bjerklöv and T. Jäger. Rotation numbers for quasiperiodically forced circle maps - Mode-locking vs strict monotonicity. J. Am. Math. Soc., 22(2):353-362, 2009. 